\title{
Oscillatory modes in an enclosed swirling flow
}

\author{
By J. M. LOPEZ ${ }^{1}$, F. MARQUES ${ }^{2}$ AND J. SANCHEZ \\ ${ }^{1}$ Department of Mathematics, Arizona State University, Tempe, AZ 85287-1804, USA \\ ${ }^{2}$ Departament de Física Aplicada, Universitat Politècnica de Catalunya, Jordi Girona Salgado s/n, \\ Mòdul B4 Campus Nord, 08034 Barcelona, Spain
}

(Received 6 July 2000 and in revised form 9 January 2001)

The flow in a completely filled cylinder driven by a rotating endwall has multiple time-dependent stable states when the endwall rotation exceeds a critical value. These states have been observed experimentally and computed numerically elsewhere. In this article, the linear stability of the basic state, which is a non-trivial axisymmetric flow, is analysed at parameter values where the unsteady solutions exist. We show that the basic state undergoes a succession of Hopf bifurcations and the corresponding eigenvalues and eigenvectors of these excited modes describe most of the characteristics of the observed time-dependent states.

\section{Introduction}

The flow in a cylinder with a rotating endwall has continued to attract much attention since Vogel (1968) first observed the vortex breakdown of the central core vortex that forms. Recent experiments (Stevens, Lopez \& Cantwell 1999) have observed a multiplicity of unsteady states that coexist over a range of the governing parameters. To date, it is not understood well where these oscillatory states originate from, how they are interrelated, nor how they are related to the steady, axisymmetric basic state.

The flow in a completely filled cylinder of radius $R$ and height $H$ is driven by the constant rotation of one endwall at angular speed $\Omega$. A schematic of the flow configuration is shown in figure 1 . The flow is governed by just two non-dimensional parameters, the aspect ratio $\Lambda=H / R$, and the Reynolds number $R e=\Omega R^{2} / v$, where $v$ is the kinematic viscosity of the incompressible fluid. Since there are just two parameters, only codimension-one or -two bifurcations can be observed. The system has one type of symmetry, invariance to rotations about the cylinder axis, generating the symmetry group $S O(2)$. For low $R e$, there is a unique branch of solutions that is steady and retains all the symmetries of the system. The only local codimensionone bifurcations that this branch can undergo are saddle-node or Hopf ones. The saddle-node of the basic state has not been observed in this system. With the Hopf bifurcation, the $S O(2)$ can either be preserved or not; both situations have been observed as $R e$ is increased in different ranges of $\Lambda$. When $S O(2)$ is preserved, the oscillatory state that results remains axisymmetric, and if $S O(2)$ is broken the result is a rotating wave (Knobloch 1994) where the axisymmetric component of the flow remains steady and a particular azimuthal mode becomes finite and precesses.

Experiments (Escudier 1984; Stevens et al. 1999) have shown that for aspect ratios $\Lambda \sim 2.5$, the basic state loses stability to an axisymmetric time-periodic state, and 


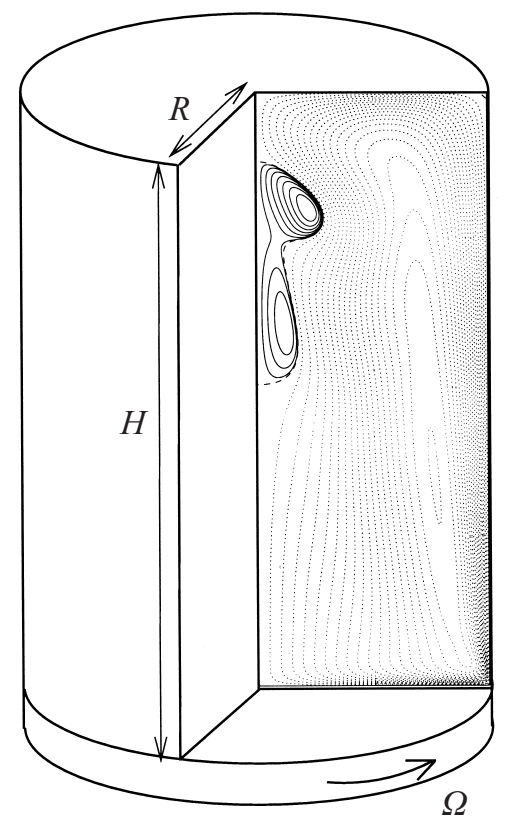

FIGURE 1. Schematic of the flow configuration.

that this bifurcation appears to be a supercritical Hopf one. Gelfgat, Bar-Yoseph \& Solan (1996) have performed a linear stability analysis of the basic state and concluded that for $\Lambda=2.5$, a supercritical Hopf bifurcation at $R e \approx 2706$ takes place. That analysis only considered axisymmetric perturbations; however Gelfgat, BarYoseph \& Solan (2001) have subsequently performed a linear stability analysis for general three-dimensional perturbations and have concluded that for $1.63<\Lambda<2.76$ the basic state loses stability via an axisymmetric Hopf bifurcation. Furthermore, Blackburn \& Lopez (2000) have computed the full three-dimensional Navier-Stokes equations for $\Lambda=2.5$ and have found that at $R e=3000$ the flow is axisymmetric and time-periodic with a period that agrees with both experiments and axisymmetric computations (Lopez 1990; Lopez \& Perry 1992; Stevens et al. 1999). At $\Lambda=2.0$, Sorensen \& Christensen (1995) have also experimentally observed that the flow, even after a Hopf bifurcation at $R e \approx 2600$, remains axisymmetric up to about $R e=3000$. So, the experiments, linear stability analysis and computations cited so far provide a consistent picture of the first bifurcation of the basic state for $\Lambda \sim 2.5$.

A recent series of experiments (Spohn, Mory \& Hopfinger 1998) provide a different picture of how the basic state loses stability. They observe steady non-axisymmetric flows at Re lower than those at which linear stability analysis (Gelfgat et al. 1996, 2001) predicts any bifurcation. Since the breaking of the $S O(2)$ symmetry always results in a rotating wave (Knobloch 1994) (of course, a stationary azimuthal wave may result at a distinguished curve in the two-dimensional parameter space, but not over a finite range of parameters as is reported in the experiments) one must conclude that either the observed stationary three-dimensional flows are a consequence of a fixed imperfection in the apparatus that destroys the $S O(2)$ symmetry, or that they arise via a non-local bifurcation that has not been detected in any other study.

Although the first bifurcation of the basic state, leading to an axisymmetric limitcycle solution for $\Lambda \sim 2.5$ is reasonably well understood, we still do not know much 
about the other unsteady states that have been observed at higher Re. These were first described by Lopez \& Perry (1992), where the axisymmetric Navier-Stokes equations were solved numerically. A similar multiplicity of unsteady states computed from the axisymmetric Navier-Stokes equations over a similar range of $\operatorname{Re}$ for $\Lambda=2.0$ have been reported by Sorensen \& Christensen (1995). Lopez \& Perry (1992) described in detail two time-periodic states with $\Lambda=2.5$, one for $R e<3500$ and another for $\operatorname{Re}>3500$. The state with $R e<3500$ corresponds to the branch that bifurcates from the basic state at $R e \approx 2700$ and a characteristic of the unsteadiness is a pulsation of the vortex breakdown bubbles with frequency $\omega_{1}$. The other state with $R e>3500$ has a qualitatively different characteristic, consisting of waves travelling on the central vortex, predominantly in the direction from the stationary endwall to the rotating endwall, with frequency $\omega_{3}$. These states were subsequently observed experimentally (Stevens et al. 1999) along with a further quasi-periodic state that exists over a range $3200<R e<3700$. This state was also computed from the axisymmetric Navier-Stokes equations and has a primary frequency $\omega_{2}$ and a lowfrequency modulation, $\omega_{\mathrm{lf}}$, two orders of magnitude smaller. The frequencies, $\omega_{1}, \omega_{2}$, and $\omega_{3}$, non-dimensionalized with the viscous time $R^{2} / v$, scale approximately as $R e$ over the range where they have been observed. In terms of the corresponding periods, Stevens et al. (1999) observed $2 \pi R e / \omega_{1} \approx 36.5,2 \pi R e / \omega_{2} \approx 57.7$, and $2 \pi R e / \omega_{3} \approx 28.8$.

The flow visualizations (Stevens et al. 1999) for $R e<3200$ appear to be essentially axisymmetric, whereas for $R e>3200$, particularly for the states with frequencies $\omega_{2}$ and $\omega_{3}$, the flows were clearly non-axisymmetric. Nevertheless, the experimentally measured periods of oscillation and their estimates from axisymmetric computations agreed uniformly to within $\approx 1 \%$. The experiments measured these oscillation periods from digitized video frames that were cross-correlated with a reference frame. Each frame consisted of an instantaneous image of the dye that was released on the axis of the stationary endwall. This dye streak was advected towards the head of the vortex breakdown bubble and subsequently spread out, so that the head of the bubble was an accumulation site for the dye. The cross-correlations, working on intensity from the laser-illuminated dye, essentially gave for each frame the displacement relative to the reference frame of the head of the bubble, which oscillated up and down the cylinder axis. So, the experiments only measured the axisymmetric component of any flow oscillations; in particular, they did not measure the precession frequency of the non-axisymmetric states.

The three time-dependent states have also been computed recently using the full three-dimensional Navier-Stokes equations (Blackburn \& Lopez 2000). They presented solutions, with $\Lambda=2.5$, on the three branches where $R e=3000\left(\omega_{1}\right.$ branch $)$, $R e=3500\left(\omega_{2}\right.$ branch), and $R e=4000$ ( $\omega_{3}$ branch). The $R e=3000$ case evolved to an axisymmetric state, whereas the other two supported rotating waves (modulated by the underlying oscillations of the axisymmetric components of the flows) with azimuthal wavenumbers $m=5$ and $m=6$, respectively. One particularly salient conclusion from that study was that the axisymmetric components of the non-axisymmetric states retain almost all $(>99 \%)$ of the kinetic energy of the flows and that the rotating waves are slaved to the axisymmetric components of the flows.

The paper is organized as follows. In $\S 2$, the axisymmetric problem is formulated in terms of the streamfunction and the axial component of angular momentum, casting the governing equations in terms of the biharmonic of the streamfunction. The numerical techniques for the solution of the nonlinear evolution, the computation of the basic state, and its linear stability analysis are presented in $\S 3$, along with a comprehensive account of the convergence properties. The results of the linear stability 


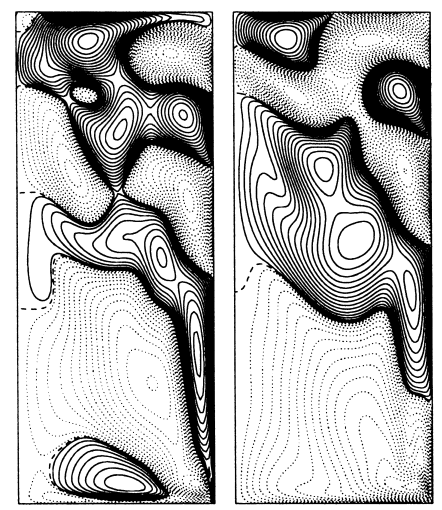

(a) $\gamma_{p}$-eigenmode, $n_{r}=70$
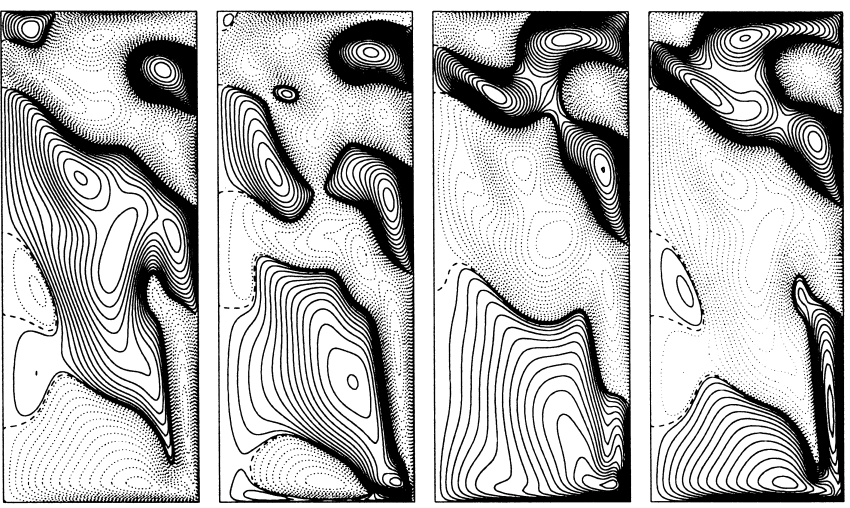

(b) $\gamma_{p}$-eigenmode, $n_{r}=140$
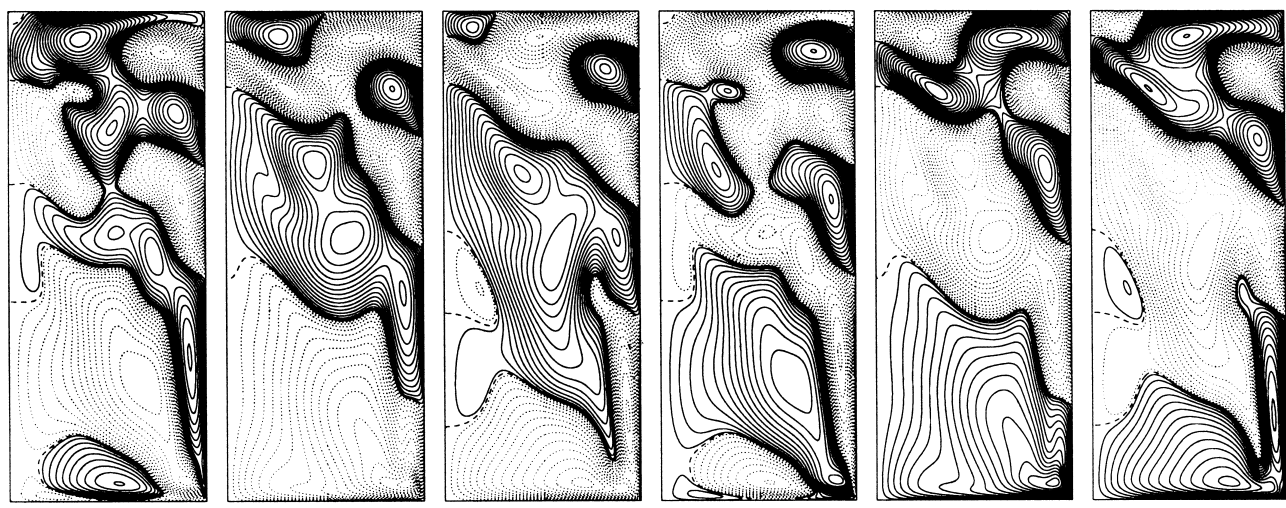

FigURE $2(a, b)$. For caption see facing page.

analysis for $\Lambda=2.5$ are given in $\S 4$, and $\S 5$ compares the linear eigenmodes with the difference between the nonlinear solutions and the basic state. These results give new insight into the origin and nature of the experimentally observed time-dependent states, which are compared with the linear modes in $\S 6$, and conclusions are drawn in section $\S 7$.

\section{Formulation}

In order to gain an understanding of the origin of the experimentally observed multiple unsteady states (Stevens et al. 1999), we wish to begin with a linear stability analysis of the steady axisymmetric basic state, determine under what conditions it undergoes Hopf bifurcations and examine if the bifurcated states are in any way related to the observed nonlinear states.

To initiate such a study, the basic state must be accurately computed. In this problem, the basic state is non-trivial, it is a function of $r$ and $z$, and an analytic description is only available in the inertialess limit $R e \rightarrow 0$, which is of no interest here since the unsteady states occur for $\operatorname{Re} \sim O\left(10^{3}\right)$. Hence, a numerical determination of the basic state is required, and this will result from the solution to the timeindependent Navier-Stokes equations restricted to an axisymmetric subspace.

For planar two-dimensional and axisymmetric problems, a convenient formulation 
(c) $\psi_{p}$-eigenmode, $n_{r}=70$
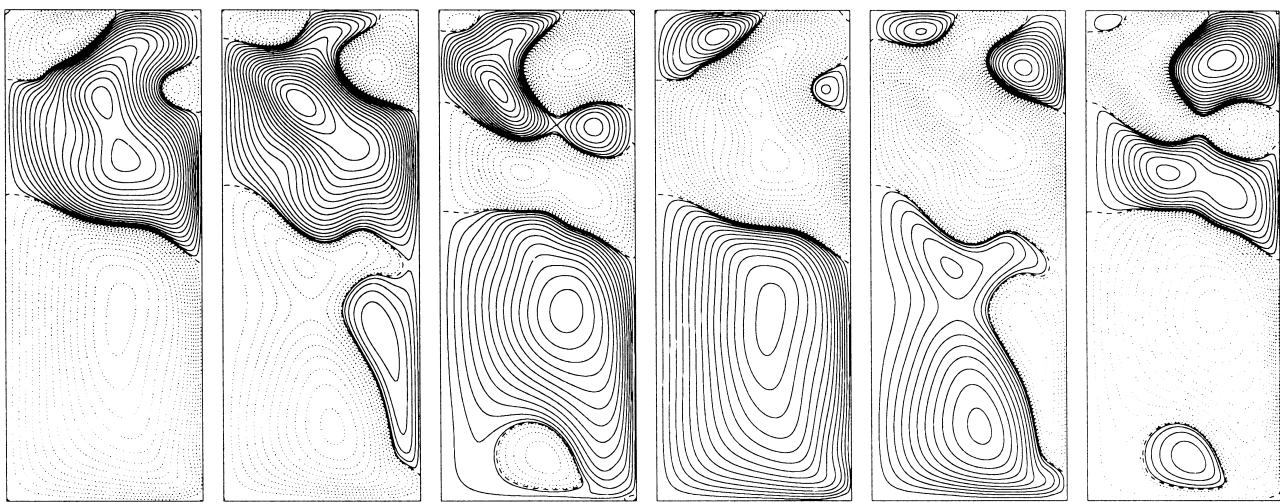

(d) $\psi_{p}$-eigenmode, $n_{r}=140$
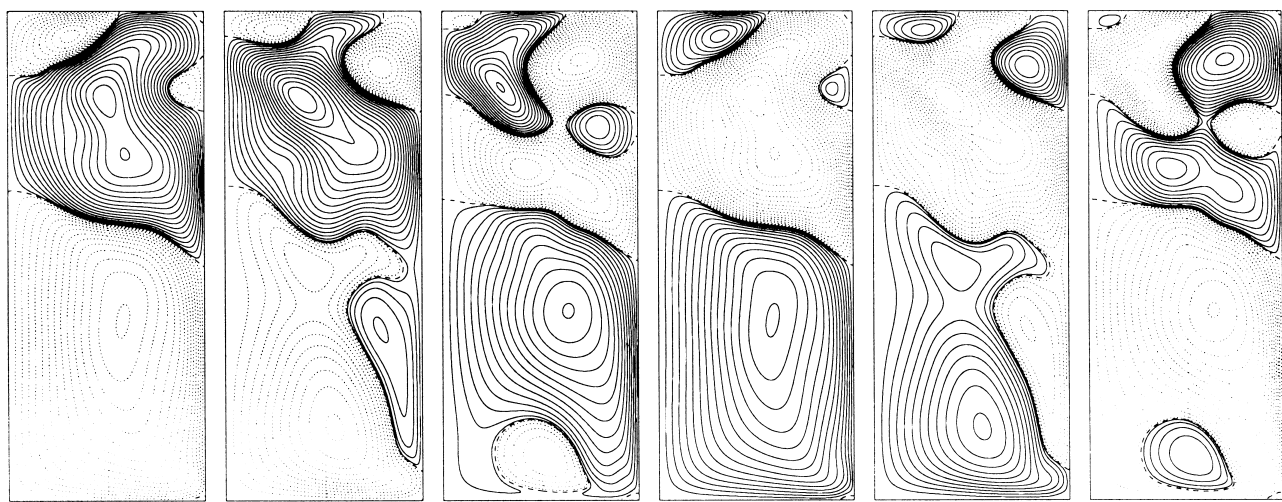

FIGURE 2. Contours of the $\gamma_{p^{-}}$and $\psi_{p}$-eigenmodes corresponding to the most dangerous eigenvalue, $\lambda_{1}$, at $\operatorname{Re}=2700, \Lambda=2.5$, over one period for $n_{r}$ as indicated.

is in terms of the streamfunction and vorticity since it is implicitly divergence-free. A drawback of this formulation is that the boundary conditions are in terms of the streamfunction and there is no explicit boundary condition for the vorticity. When this formulation is used for nonlinear evolution, this problem with the boundary conditions is overcome by using an explicit temporal scheme, but for time-independent solutions and linear stability analysis this is not appropriate. In these cases, one would like an implicit solution of the Laplace operators, and this requires explicit implementation of the boundary conditions.

One way to resolve the boundary condition problem is to treat the vorticity only as an auxiliary variable and formulate the problem in terms of the streamfunction, leading to a biharmonic formulation. Our problem is a little more involved due to the rotation of the bottom endwall which introduces an additional variable, the azimuthal component of velocity, $v$, which we shall incorporate in terms of the (axial) angular momentum, $\gamma=r v$. In cylindrical polar coordinates, the velocity and vorticity vectors are

$$
\boldsymbol{u}=\left(-\psi_{z}, \gamma, \psi_{r}\right) / r \text { and } \boldsymbol{\nabla} \times \boldsymbol{u}=\left(-\gamma_{z}, r \eta, \gamma_{r}\right) / r,
$$

where $\psi$ is the streamfunction and $\eta$, the azimuthal component of vorticity, is 

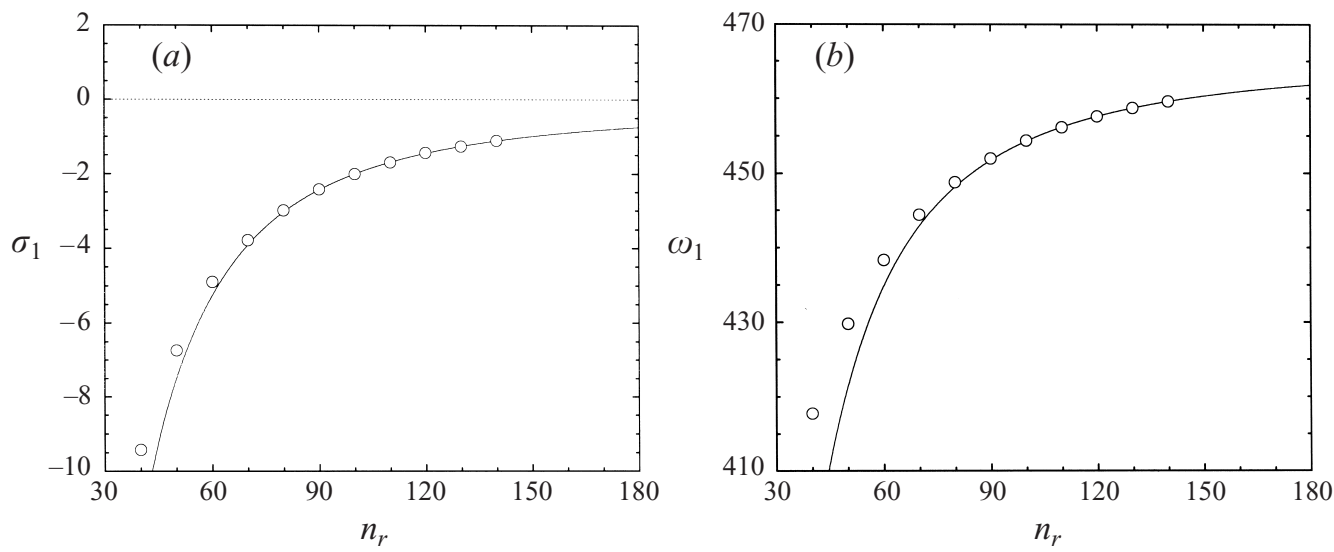

FIGURE 3. Variations of $(a) \sigma_{1}$ and $(b) \omega_{1}$ of the most dangerous eigenvalue with $n_{r}$, for $R e=2700$ and $\Lambda=2.5$. The solid lines are fits of the form $a+b / r^{2}$ for $n_{r} \geqslant 110$.

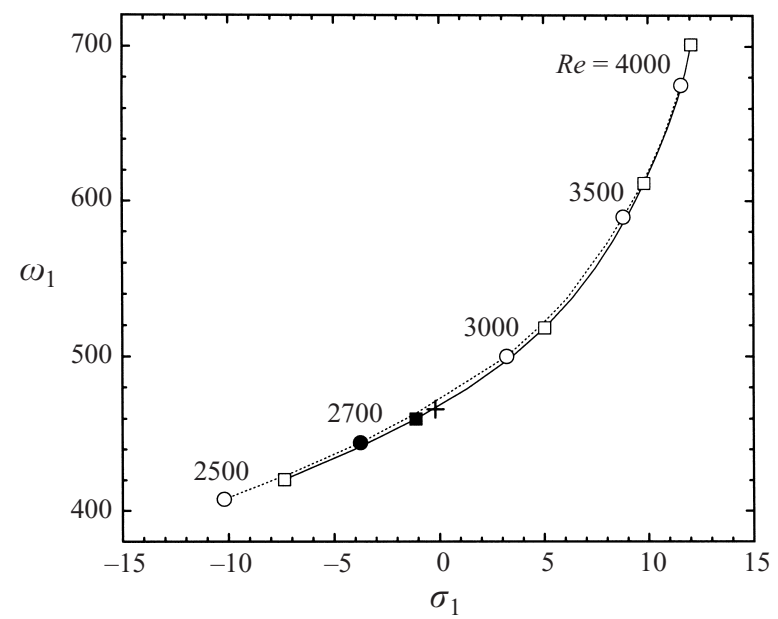

FigURE 4. Eigenvalues on branch $1, \lambda_{1}=\sigma_{1}+\mathrm{i} \omega_{1}$, evaluated with $n_{r}=70$ (dashed curve and circles) and $n_{r}=140$ (solid curve and squares) over a range of $R e \in[2500,4000]$ with $\Lambda=2.5$. The asymptotic estimate at $R e=2700$ is the plus symbol.

related to $\psi$ via

$$
\Delta_{*} \psi=\psi_{z z}+\psi_{r r}-\psi_{r} / r=-r \eta .
$$

Note that contours of $\psi$ in an $(r, z)$-plane depict the streamlines of the flow, and likewise contours of $\gamma$ depict the vortex lines. The governing equations, nondimensionalized using $R$ as the length scale and $R^{2} / v$ as the time scale, are the azimuthal components of the Navier-Stokes equations and their curl:

$$
\left.\begin{array}{l}
\partial_{t} \gamma=\Delta_{*} \gamma+(1 / r) \psi_{z} \gamma_{r}-(1 / r) \psi_{r} \gamma_{z}, \\
\partial_{t} \Delta_{*} \psi=\Delta_{*}^{2} \psi+(1 / r) \psi_{z} \partial_{r}\left(\Delta_{*} \psi\right)-(1 / r) \psi_{r} \Delta_{*} \psi_{z}-\left(2 / r^{2}\right) \psi_{z} \Delta_{*} \psi-\partial_{z}\left(\gamma^{2} / r^{2}\right) .
\end{array}\right\}
$$

The governing equations can be written in a more compact form using $\boldsymbol{x}=(\gamma, \psi)$ :

$$
\partial_{t} \boldsymbol{B} \boldsymbol{x}=\boldsymbol{L} \boldsymbol{x}+\boldsymbol{N}(\boldsymbol{x}, \boldsymbol{x})=f(\boldsymbol{x}), \quad \boldsymbol{B}=\left(\begin{array}{cc}
1 & 0 \\
0 & \Delta_{*}
\end{array}\right), \quad \boldsymbol{L}=\left(\begin{array}{cc}
\Delta_{*} & 0 \\
0 & \Delta_{*}^{2}
\end{array}\right),
$$




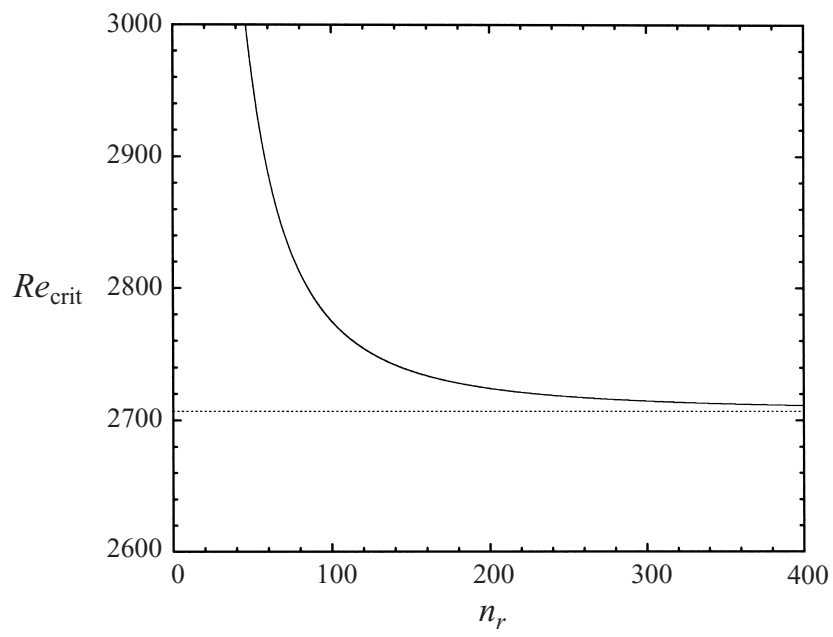

FIGURE 5. Variation of $R e_{\text {crit }}$ with $n_{r}$, obtained using the fit (4.1) which is valid in the asymptotic regime $\left(n_{r} \geqslant 90\right)$.

and $\boldsymbol{N}$ is the quadratic nonlinearity. The corresponding boundary conditions are $\psi=\psi_{r}=\gamma=0$ on the axis $(r=0)$, the cylinder wall $(r=1)$, and the top endwall $(z=\Lambda) ; \psi=\psi_{r}=0, \gamma=\operatorname{Rer}^{2}$ on the bottom rotating endwall $(z=0)$.

To solve for the basic state, $\boldsymbol{x}_{b}=\left(\gamma_{b}, \psi_{b}\right)$, the left-hand side of (2.2) is set to zero. The linear stability of the basic state is determined by linearizing (2.2) about the basic state, with

$$
\boldsymbol{x}(r, z, t)=\boldsymbol{x}_{b}(r, z)+\mathrm{e}^{\lambda t} \boldsymbol{x}_{p}(r, z),
$$

where the perturbation $\boldsymbol{x}_{p}$ satisfies homogeneous boundary conditions. The linearized equations for $\boldsymbol{x}_{p}$ lead to the generalized eigenvalue problem

$$
\lambda \boldsymbol{B} \boldsymbol{x}_{p}=\boldsymbol{L} \boldsymbol{x}_{p}+\boldsymbol{N}\left(\boldsymbol{x}_{b}, \boldsymbol{x}_{p}\right)+\boldsymbol{N}\left(\boldsymbol{x}_{p}, \boldsymbol{x}_{b}\right)=\boldsymbol{D} f\left(\boldsymbol{x}_{b}\right) \boldsymbol{x}_{p},
$$

where $\boldsymbol{D} f\left(\boldsymbol{x}_{b}\right)$ is the Jacobian of the right-hand side of (2.2) evaluated at $\boldsymbol{x}_{b}$.

\section{Numerical techniques}

Edwards et al. (1994) and Tuckerman \& Barkley (2000) advocate the use of a single suite of routines for the nonlinear evolution, the steady-state solver, and linear stability analysis. This is the type of approach that we also employ. We shall begin with a description of the nonlinear evolution solver as it provides the basic building blocks for the complete package.

\subsection{Nonlinear evolution solver}

The time derivatives in (2.2) are approximated by a second-order backwards timedifference, the linear term is treated implicitly, and a second-order backwards extrapolation scheme is used for the nonlinear term, leading to

$$
\left(\frac{3}{2 \delta t} \boldsymbol{B}-\boldsymbol{L}\right) \boldsymbol{x}^{k}=\frac{2}{\delta t} \boldsymbol{B} \boldsymbol{x}^{k-1}-\frac{1}{2 \delta t} \boldsymbol{B} \boldsymbol{x}^{k-2}+2 \boldsymbol{N}\left(\boldsymbol{x}^{k-1}, \boldsymbol{x}^{k-1}\right)-\boldsymbol{N}\left(\boldsymbol{x}^{k-2}, \boldsymbol{x}^{k-2}\right),
$$

where $\delta t$ is the time step and $\boldsymbol{x}^{k}=\boldsymbol{x}\left(t_{0}+k \delta t\right)$.

Discretization in space is accomplished using second-order centred differences. 


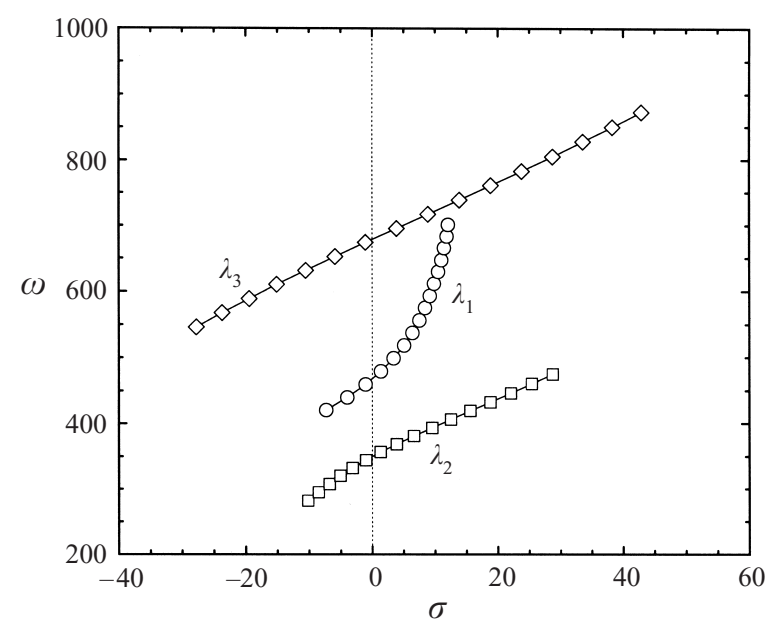

FIGURE 6. Real and imaginary parts of the three most dangerous eigenvalues for $\Lambda=2.5$ and $2500 \leqslant R e \leqslant 4000$, using $n_{r}=140$.

(a) $\gamma_{b}$

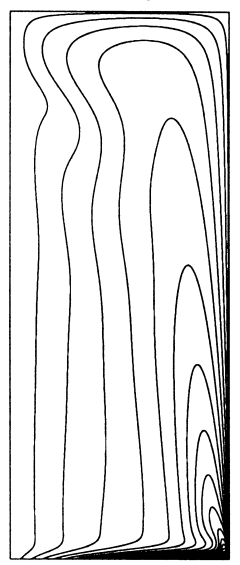

(b) $\psi_{b}$

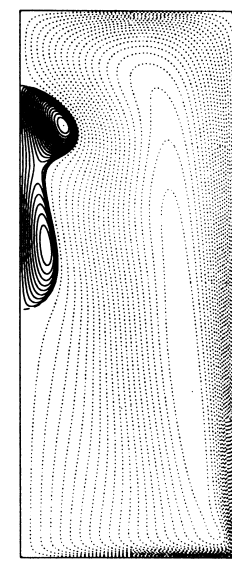

(c) $v_{b}$

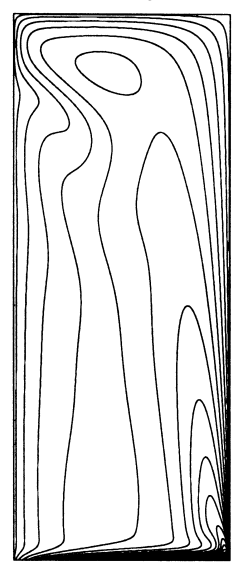

(d) $w_{b}$

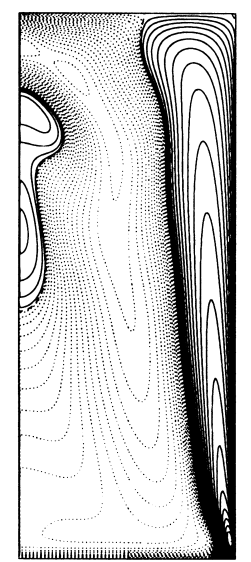

FIGURE 7. Contours of $\gamma_{b}, \psi_{b}, v_{b}$, and $w_{b}$ for $\operatorname{Re}=2700, \Lambda=2.5, n r=140$.

The most critical aspect is the efficient solution of the linear operator acting on $\boldsymbol{x}^{k} . \boldsymbol{L}$ consists of two operators, $\Delta_{*}$ and $\Delta_{*}^{2}$. For the single Laplacian $\Delta_{*}$, we simply diagonalize in the $z$-direction and obtain a system of one-dimensional solutions in $r$. For the biharmonic,

$$
\Delta_{*}^{2}=\partial_{r r r r}-(2 / r) \partial_{r r r}+\left(3 / r^{2}\right) \partial_{r r}-\left(3 / r^{3}\right) \partial_{r}+\left(2 \partial_{r r}-(2 / r) \partial_{r}\right) \partial_{z z}+\partial_{z z z z},
$$

the $\partial_{z z}$ and $\partial_{z z z z}$ operators cannot be diagonalized simultaneously. The $\partial_{z z z z}$ operator is almost the square of $\partial_{z z}$, but it is not exactly so due to the need to use one-sided differences in $\partial_{z z z z}$ at the boundaries. However, the banded structure from the finite difference discretization still allows for an efficient direct solver.

\subsection{Steady-state solver}

The time-independent governing equations for the basic state, $x_{b}$, are

$$
f(x)=L x+N(x, x)=0,
$$


(a) $\gamma_{p}$-eigenmode, mode 2
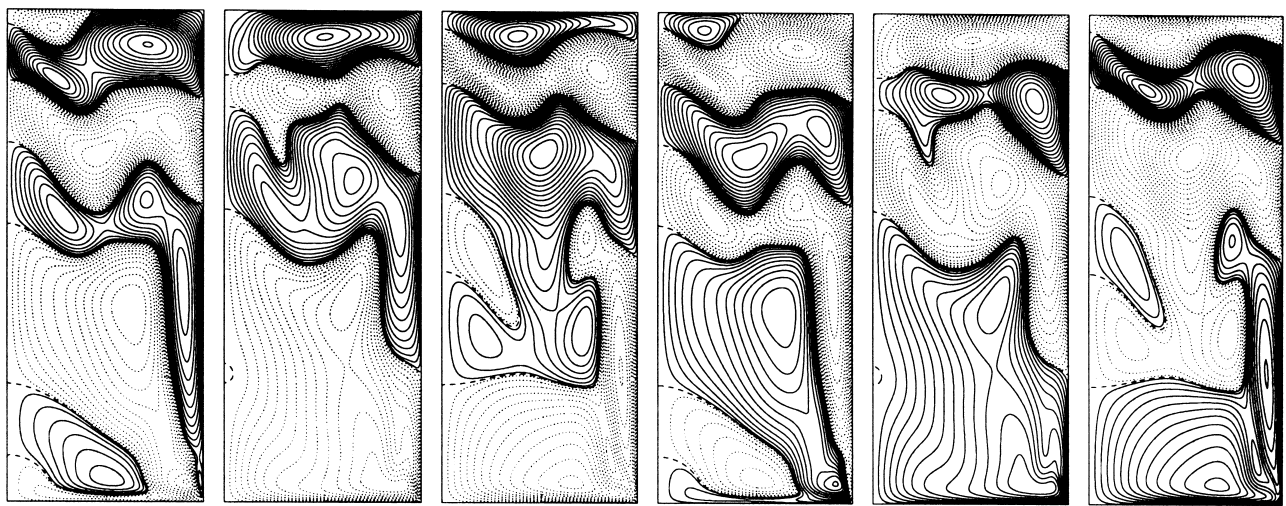

(b) $\gamma_{p}$-eigenmode, mode 3
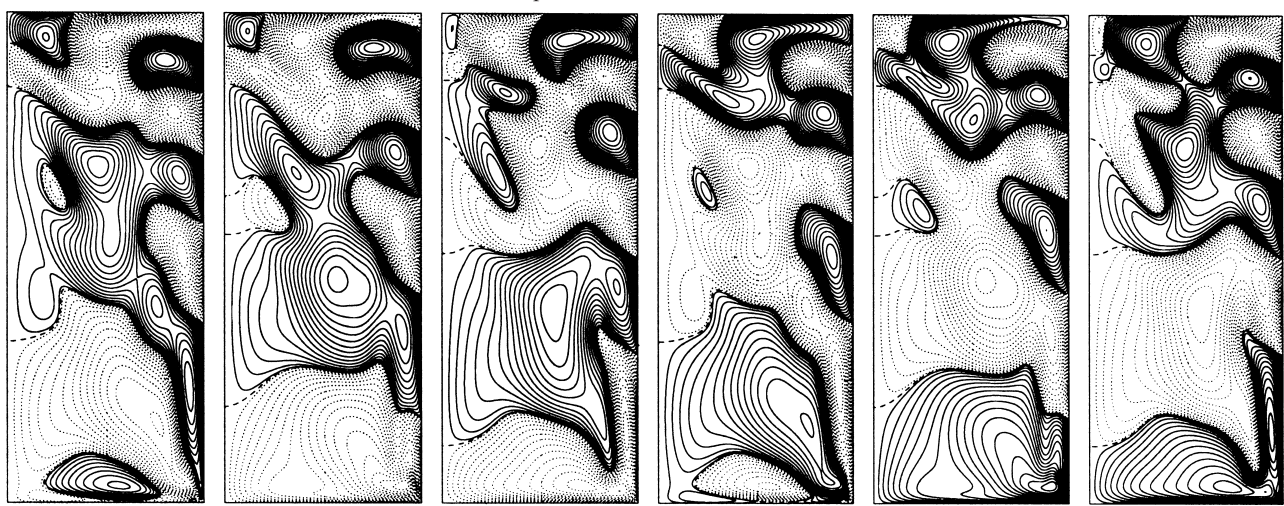

FIGURE 8. Contours of the $\gamma_{p}$-eigenmodes corresponding to eigenvalues $\lambda_{2}$ and $\lambda_{3}$ at $R e=2700$, $\Lambda=2.5$, over one period.

where $\boldsymbol{L}$ and $\boldsymbol{N}$ are discretized as in $\S 3.1$. This equation is solved by Newton iteration

$$
\boldsymbol{D} f\left(\boldsymbol{x}_{b}^{i}\right)\left(\boldsymbol{x}_{b}^{i+1}-\boldsymbol{x}_{b}^{i}\right)=-f\left(\boldsymbol{x}_{b}^{i}\right),
$$

where $\boldsymbol{D} f\left(\boldsymbol{x}_{b}^{i}\right)$ acting on a vector $\boldsymbol{y}$ is

$$
\boldsymbol{D} f\left(\boldsymbol{x}_{b}^{i}\right) \boldsymbol{y}=\boldsymbol{L} \boldsymbol{y}+\boldsymbol{N}\left(\boldsymbol{x}_{b}^{i}, \boldsymbol{y}\right)+\boldsymbol{N}\left(\boldsymbol{y}, \boldsymbol{x}_{b}^{i}\right) .
$$

For an efficient solution of (3.1), two things are required: a good initial guess $\boldsymbol{x}_{b}^{0}$ and an efficient linear solver for (3.2). By using continuation from $R e=0$, we always have a good estimate of $\boldsymbol{x}_{b}^{0}$ for subsequently larger $R e$. Due to the size of the Jacobian, direct methods are impractical, and so we use a matrix-free iterative algorithm based on Krylov subspaces, known as generalized minimal residuals, GMRES (Trefethen $\&$ Bau 1997). To accelerate the convergence of GMRES it must be appropriately preconditioned; we use $\boldsymbol{L}$ as the preconditioner.

\subsection{Linear stability}

The linear stability of the basic state, $\boldsymbol{x}_{b}$, is determined by the eigenvalue from (2.3) with largest real part (growth rate). If its real part is positive (negative), the basic state is unstable (stable). To solve the eigenvalue problem (2.3) we use the Arnoldi method as implemented in the ARPACK package (Lehoucq, Sorensen \& Yang 1998). This 

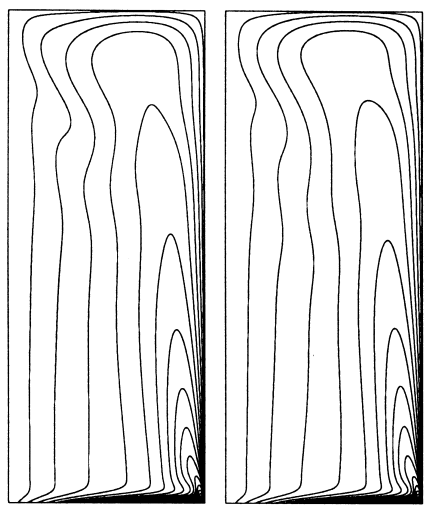

(a) $\gamma, \operatorname{Re}=3000$
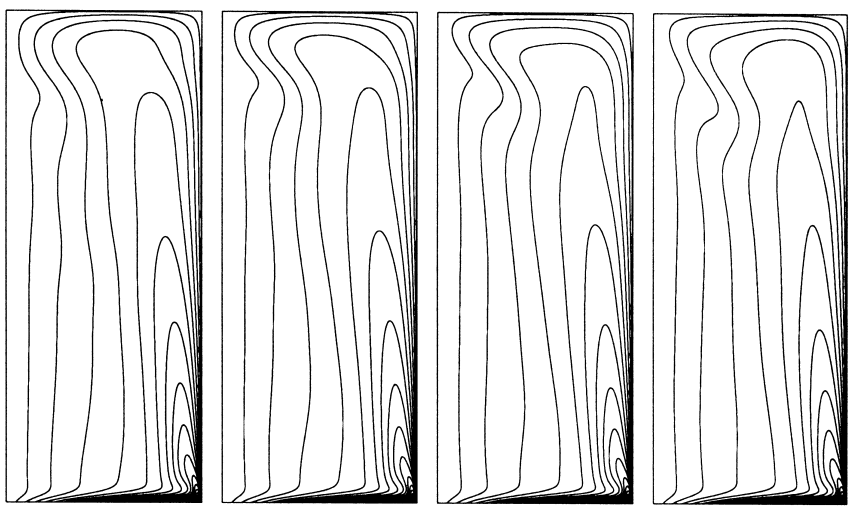

(b) $\psi, R e=3000$
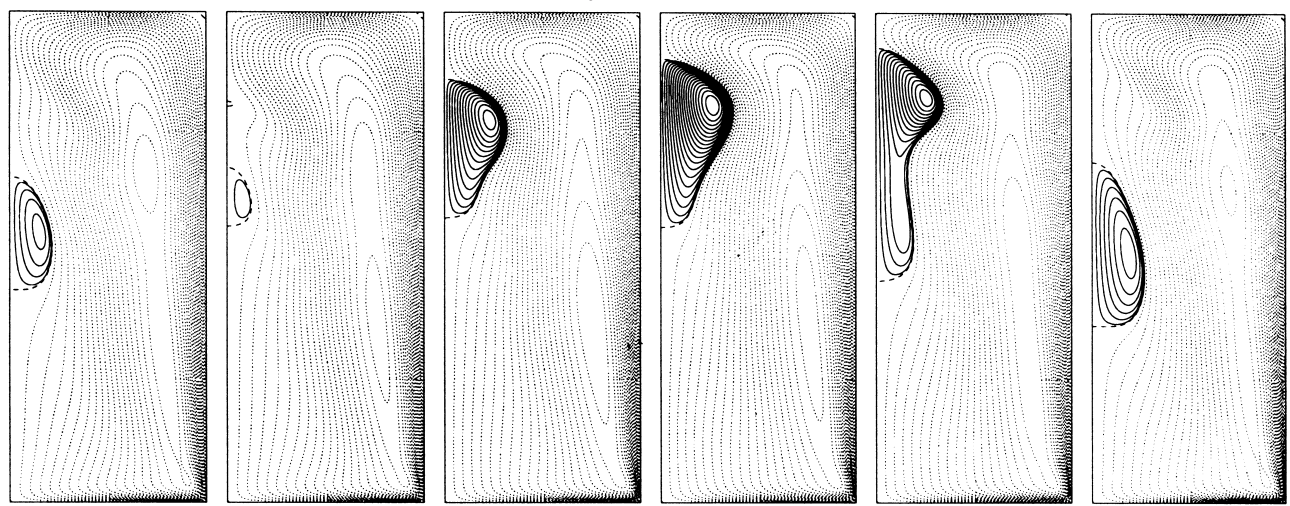

FigURE $9(a, b)$. For caption see facing page.

iterative technique is based on the power method, where the eigenvalues of a subspace generated by the powers of an initial vector are found. The method converges to the eigenvalues with largest moduli. Since we are interested in the eigenvalues with largest real part, rather than largest moduli, we implement a Cayley transformation converting (2.3) to

$$
\left(\boldsymbol{D} f\left(\boldsymbol{x}_{b}\right)-\beta \boldsymbol{B}\right)^{-1}\left(\boldsymbol{D} f\left(\boldsymbol{x}_{b}\right)-\alpha \boldsymbol{B}\right) \boldsymbol{x}_{p}=\tilde{\lambda} \boldsymbol{x}_{p} .
$$

The eigenvalues of (3.3) are related to those of (2.3) by

$$
\tilde{\lambda}=\frac{\lambda-\alpha}{\lambda-\beta},
$$

where $\alpha$ and $\beta$ are real in our implementation. On using the Arnoldi method (3.3) converges to eigenvalues of largest moduli, and with suitably chosen $\alpha$ and $\beta$ these correspond to the eigenvalues of (2.3) with largest real part, through (3.4). The eigenvectors of (3.3) and (2.3) are the same.

\subsection{General considerations}

The most critical aspect in all three types of solvers listed above involves the solution of large linear systems of the form

$$
(\boldsymbol{A}+c \boldsymbol{B}) \boldsymbol{y}=\boldsymbol{a},
$$



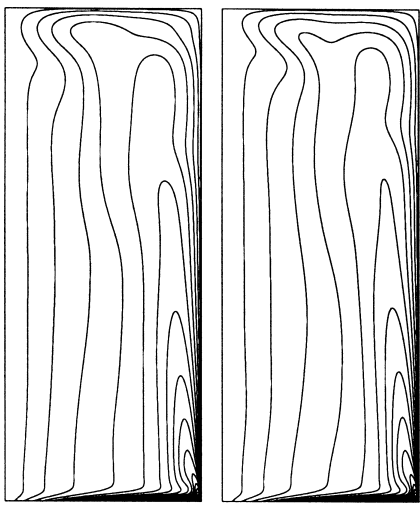

(c) $\gamma, \operatorname{Re}=4000$
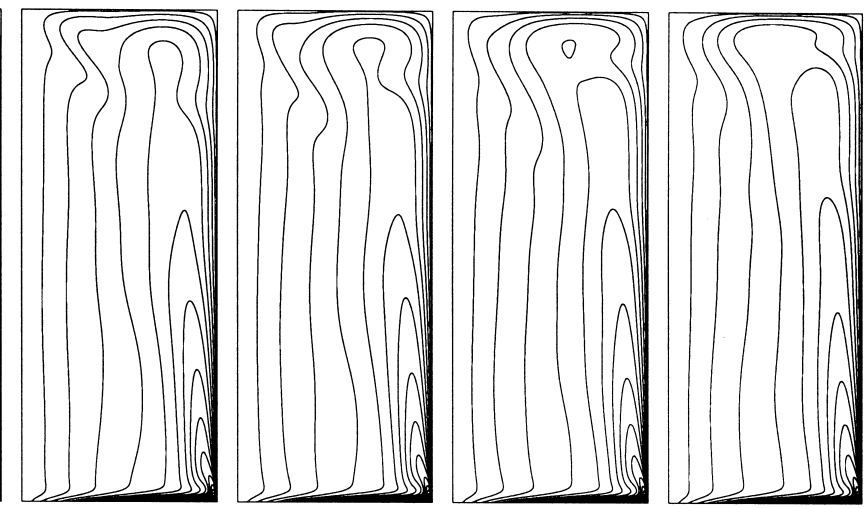

(d) $\psi, R e=4000$
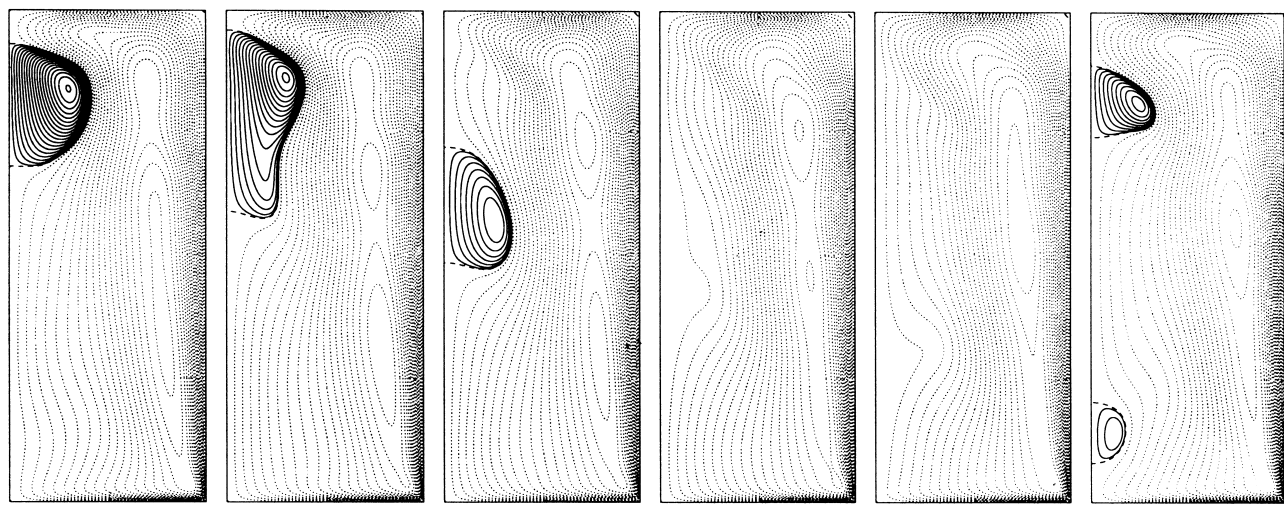

Figure 9. Contours of $\gamma$ and $\psi$ over one period for $R e=3000$ and 4000, $\Lambda=2.5, n_{r}=140$.

where $\boldsymbol{a}$ is known. The three types of solvers differ in the specification of $A$ and $c$ :

$$
\begin{array}{lll}
\text { Nonlinear evolution } & \boldsymbol{A}=\boldsymbol{L}, & c=-3 / 2 \delta t, \\
\text { Steady-state solver } & \boldsymbol{A}=\boldsymbol{D} f\left(\boldsymbol{x}_{b}^{i}\right), \quad c=0, \\
\text { Linear stability } & \boldsymbol{A}=\boldsymbol{D} f\left(\boldsymbol{x}_{b}\right), \quad c=-\beta .
\end{array}
$$

For the nonlinear evolution, (3.5) is solved directly. For the steady-state solver and linear stability analysis, (3.5) is solved via GMRES with $\boldsymbol{L}$ as the preconditioner, which comes directly from the nonlinear evolution solver.

\subsection{Convergence with spatial resolution}

The most sensitive physical feature of the flow to be resolved is the Ekman boundary layer on the rotating endwall, whose thickness scales with $R e^{-1 / 2}$. The convergence of the eigenvalue problem is by far the most sensitive computation to grid resolution. Throughout, we use a uniform grid with $n_{z} / n_{r}=\Lambda$, where $n_{r}$ and $n_{z}$ are the number of grid intervals in the radial and axial directions. We now compare the $\gamma_{p^{-}}$and $\psi_{p}$-eigenmodes corresponding to the most dangerous eigenvalue, i.e. that with largest real part, for $R e=2700$ and $\Lambda=2.5$, computed with $n_{r}=70$ and $n_{r}=140$. In fact, this eigenvalue is one of a pair of complex conjugates, and so the eigenvector has a harmonic temporal dependence. For the eigenvalue pair $\sigma_{1} \pm \mathrm{i} \omega_{1}$, the corresponding 
eigenvectors are $y_{R} \pm \mathrm{i} y_{I}$. The corresponding mode has the form

$$
\operatorname{Re}\left(\mathrm{e}^{\left(\sigma_{1}+\mathrm{i} \omega_{1}\right) t}\left(y_{R}+\mathrm{i} y_{I}\right)\right)=\mathrm{e}^{\sigma_{1} t}\left(y_{R} \cos \omega_{1} t-y_{I} \sin \omega_{1} t\right) .
$$

Figure 2 shows six equally spaced phases of $y_{R} \cos \omega_{1} t-y_{I} \sin \omega_{1} t$ over one period $\tau_{1}=2 \pi / \omega_{1}$. For $n_{r}=70, \omega_{1}=444.4$, and for $n_{r}=140, \omega_{1}=459.6$. Note that the complex eigenvectors are determined up to an arbitrary phase. In the figure, we have adjusted the phase up to $\pi / 30$ in order to synchronize the oscillations between the $n_{r}=70$ and $n_{r}=140$ cases. From this figure, we see that there is very little difference in either the temporal or spatial structure of this most dangerous mode, and so with $n_{r}=70$ all salient features of the flow are accurately captured. To obtain a more quantitative measure of the convergence with increasing $n_{r}$, we examine how $\sigma_{1}$ and $\omega_{1}$ vary with $n_{r}$.

In figure $3, \sigma_{1}$ and $\omega_{1}$ for $R e=2700$ and $\Lambda=2.5$ are plotted for $n_{r} \in[40,140]$ in steps of 10 . We see from this figure that for $n_{r} \geqslant 90$, the system is well within the asymptotic regime where the computed $\sigma_{1}$ and $\omega_{1}$ converge as $n_{r}^{-2}$. This quadratic convergence is to be expected from our second-order scheme. The fits shown in the figure are

$$
\lambda_{1}\left(n_{r}\right)=-0.18454+465.167 \mathrm{i}-(18230+108912 \mathrm{i}) / n_{r}^{2} .
$$

This allows us to estimate the asymptotic values $\lim _{n_{r} \rightarrow \infty} \lambda_{1}=-0.18454+465.167 \mathrm{i}$. Compared with the value at $n_{r}=140,-1.1159+459.617 \mathrm{i}$, we have a relative error of $1.2 \%$. Furthermore, this error is not simply randomly oriented in the complex plane. Figure 4 shows the variation of this eigenvalue with $R e$ for $n_{r}=70$ and $n_{r}=140$, together with the asymptotic estimate at $R e=2700$. Although there are variations due to $n_{r}$ in the location of the eigenvalues in the complex plane, they are located on a well-defined curve. In essence, computations of eigenvalues using a relatively low $n_{r}$ correspond to a precise computation at an effectively lower $R e$. This phenomenon can be traced back, in part, to the degree to which the Ekman layer can be resolved with finite $n_{r}$, and to artifical viscosity introduced by the numerics on the coarse grid throughout the domain.

In the nonlinear evolution of the axisymmetric Navier-Stokes equations, with $\Lambda=2.0$, Sorensen \& Christensen (1995) observed with their uniformly spaced finite difference grid that except for a displacement of the Reynolds numbers, the general behaviour of the transition scenario did not depend critically on the grid resolution. They concluded that $n_{r} \sim 100$ is sufficient to capture qualitatively correct dynamics. In all the results presented in our study, $n_{r}=140$ has been used.

\section{Results of the linear stability analysis}

We begin by determining the critical $R e$ at which the basic state first loses stability. We shall restrict our investigation to the $\Lambda=2.5$ case since this is the aspect ratio used in the experiments of Stevens et al. (1999).

\subsection{Onset of unsteadiness}

The basic state, $x_{b}$, is computed using $n_{r}=140$, and the sixteen eigenvalues with largest real part are found over a range of Re. Using this spatial resolution, $x_{b}$ loses stability as a pair of complex conjugate eigenvalues cross the imaginary axis at $R e \approx 2742$. We can estimate the critical $R e$ for this Hopf bifurcation in the limit $n_{r} \rightarrow \infty$ using the results of $\S 3.5$. For $R e=2700, \sigma_{1}=-0.18454-18230 / n_{r}^{2}$ and for $R e=2710, \sigma_{1}=0.077777-18163 / n_{r}^{2}$. Using linear interpolation, the critical $R e$ as a 


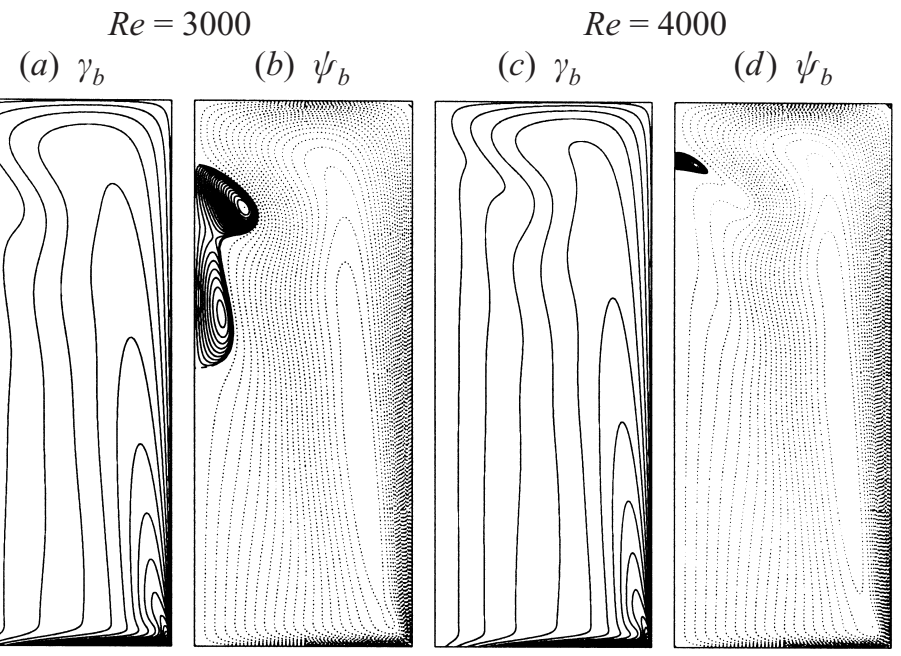

Figure 10. Contours of $\gamma_{b}$ and $\psi_{b}$ for $R e=3000$ and 4000, $\Lambda=2.5, n_{r}=140$.

function of $n_{r}$ is

$$
R e_{\text {crit }}=2707+\frac{2722}{1+n_{r}^{2} / 254.6},
$$

giving the asymptotic estimate $R e_{\text {crit }}=2707$. The relative error in $R e_{\text {crit }}$ using $n_{r}=140$ is $1.4 \%$. Figure 5 is a plot of the expression (4.1), together with the asymptotic value (dashed line). This asymptotic value agrees quite well with the estimate of Gelfgat et al. (1996) from their linear stability analysis $\left(R e_{\text {crit }} \approx 2706\right)$, and the experimental estimates of $R e_{\text {crit }} \approx 2700$ to within about $1 \%$ (Escudier 1984; Stevens et al. 1999). The Hopf frequency, $\omega_{1}$, also compares very well with the experiments and the linear stability analysis of Gelfgat et al. (1996); we obtain $\omega_{1} \approx 460$ and the other studies obtain $0.17 R e \approx 460$ (we have used $R^{2} / v$ to scale time whereas they used $\Omega$, hence the $R e$ factor).

\subsection{Subsequent Hopf bifurcations of the basic state}

There are three nonlinear unsteady branches that are observed experimentally. In this section, we examine in some detail the three most dangerous eigenvalues of the basic state. These are plotted in figure 6 , where they have been computed over the range $2500 \leqslant R e \leqslant 4000$ in increments of 100 , depicting three distinct branches, labelled 1,2 , and 3 according to the order in which they cross the imaginary axis as $R e$ is increased. As we shall see, these three modes of the basic state capture the essential features of the nonlinear states. Even though the second and third modes are unstable limit cycles when they bifurcate from the unstable basic state, they play an important role as organizing centres for the observed nonlinear dynamics.

Before exploring the spatial structure of the perturbation modes $x_{p}$, it is useful to re-examine the spatial structure of the basic state. Figures $7(a)$ and $7(b)$ show $\gamma_{b}$ and $\psi_{b}$ for $R e=2700, \Lambda=2.5$. For this flow driven by the rotating endwall, the azimuthal velocity is the primary flow component, represented here by $\gamma_{b}$ whose contours give the vortex lines. All the vortex lines emanate from the rotating endwall and terminate at the corner where the rotating endwall and the stationary sidewall meet. This basic feature of the flow, present for all $R e$, necessitates the bending of the vortex lines, producing meridional flow, $\psi_{b}$, via the $\partial_{z}\left(\gamma^{2} / r^{2}\right)$ term in (2.1). For 

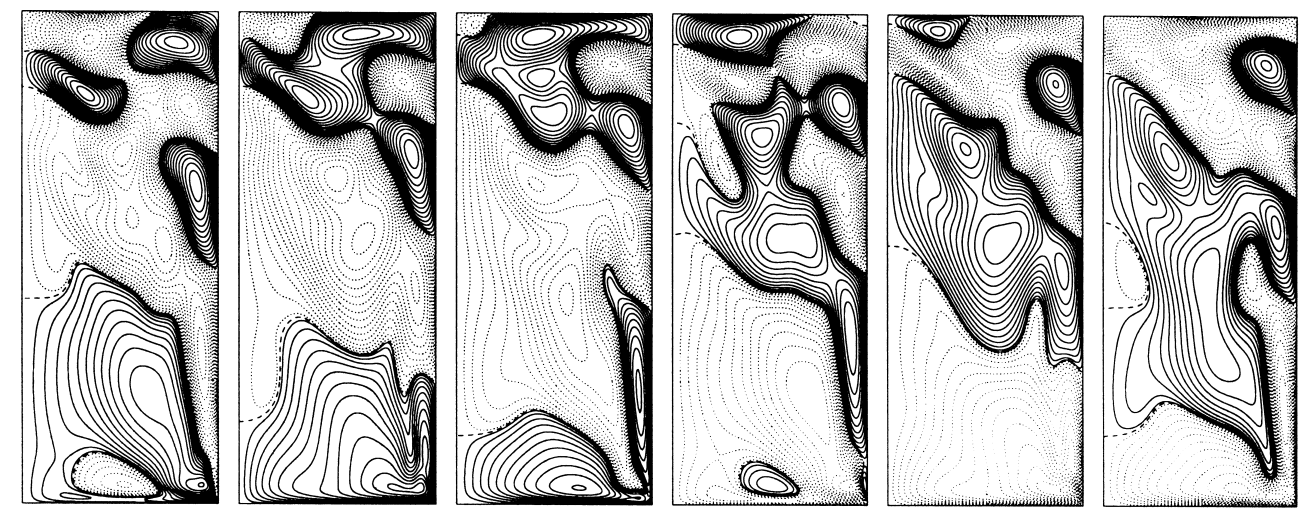

(b) $\gamma_{p}$-eigenmode, $R e=3000$
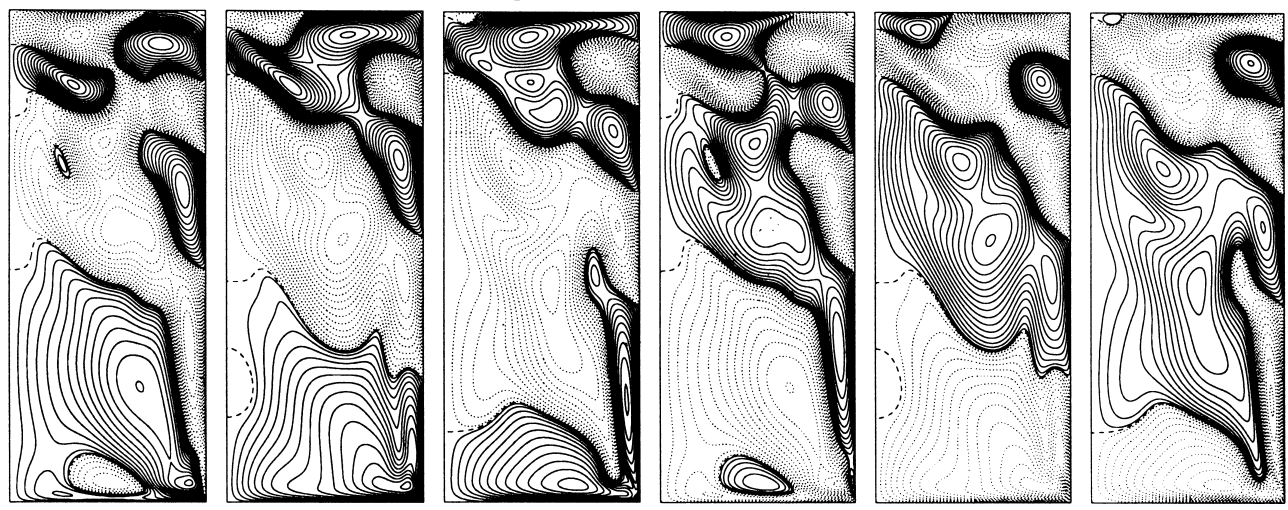

FIGURE $11(a, b)$. For caption see facing page.

a description of the physical mechanism involving vortex line bending responsible for the recirculation zones in $\psi_{b}$ on the axis, see Brown \& Lopez (1990). As Re is increased, the secondary meridional flow is intensified, further bending the vortex lines and producing an Ekman layer on the rotating disk whose thickness scales with $R e^{-1 / 2}$. Due to the presence of the sidewall, the Ekman layer is turned into the axial direction and a swirling jet-like structure results near the sidewall. The locus of the jet's maximum $\gamma_{b}$ at each axial station makes an angle of approximately $6^{\circ}$ with the sidewall. As we shall see, the linear perturbation modes from the stability analysis of the basic state are primarily related to this jet.

Figures $7(c)$ and $7(d)$ show contours of the azimuthal and axial velocities of the basic state, $v_{b}$ and $w_{b}$. The contours of $v_{b}$ are similar to the vortex lines, but the position of the maximum (the jet core) is tilted to the interior with respect to the maximum in angular momentum (the angle for the $v_{b}$-jet is approximately $9^{\circ}$ ). The $\gamma_{b}$-jet core is located very close to the zero-axial-velocity curve $\left(w_{b}\right)$, separating flow moving up the sidewall from the return flow to the rotating disk in the interior. Even though there are shear instability modes associated with the axial velocity profile, the azimuthal velocity is an order of magnitude larger and the most dangerous modes of the basic state correspond to instabilities of the azimuthal velocity profile. In particular, (negative) gradients in $\gamma_{b}$ are greatest on the sidewall side of the jet. These gradients are centrifugally unstable and contribute to the most dangerous modes 

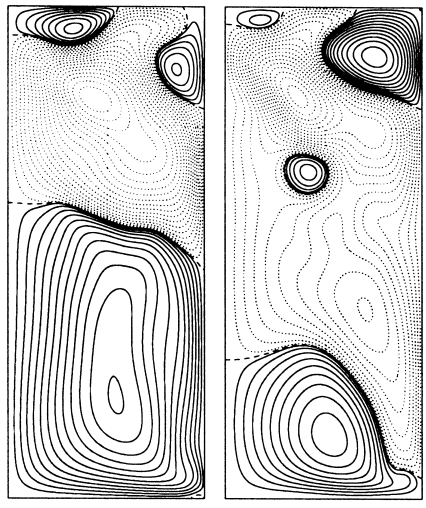

(c) $\psi-\psi_{b}, R e=3000$
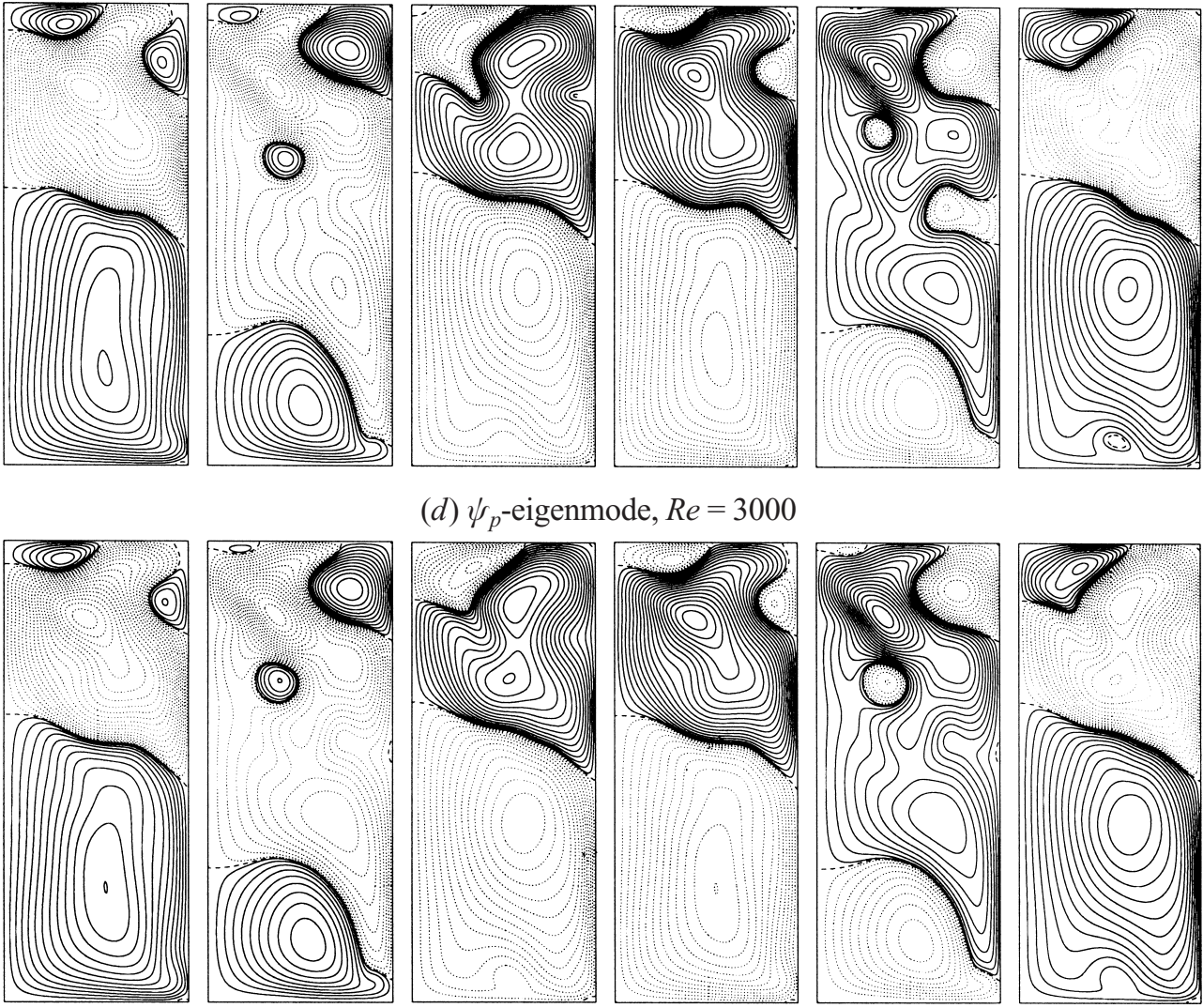

(d) $\psi_{p}$-eigenmode, $R e=3000$
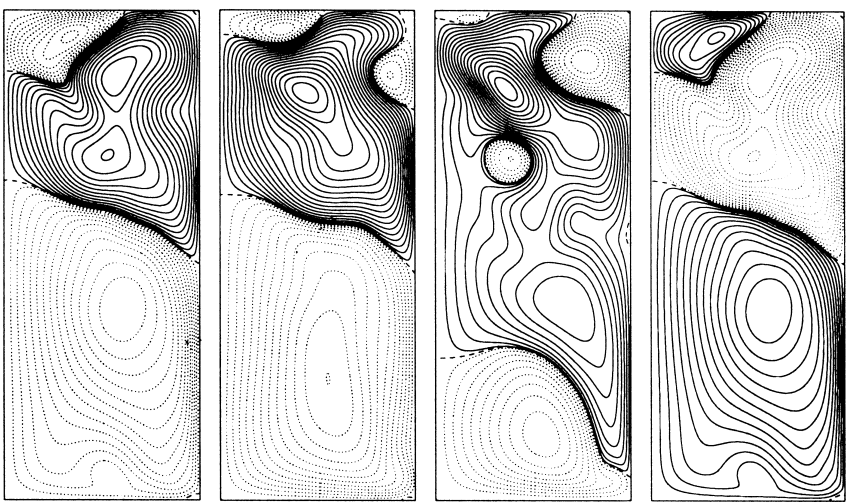

FIGURE 11. Contours of $(a) \gamma-\gamma_{b}$ and $(c) \psi-\psi_{b}$ over one nonlinear period $1.217 \times 10^{-2}$ and $(a) \gamma_{p^{-}}$ and $(c) \psi_{p}$-eigenmodes of $\lambda_{1}$ over one period $\tau_{1}=2 \pi / \omega_{1}=1.213 \times 10^{-2}$ for $R e=3000, \Lambda=2.5$, $n_{r}=140$

of the basic state. Figures $2(b)$ and 8 show the $\gamma_{p}$-eigenmodes corresponding to the three most dangerous eigenvalues. The most prominent characteristic of these modes is a series of cellular structures located between the jet core and the sidewall that travel up the sidewall and turn in towards the interior at the stationary endwall. Different modes differ in the number of such structures. The drift axial velocity of these structures is closely related to the axial velocity of the basic state in this region. These cellular structures are reminiscent of Görtler vortices with a mean drift in the crossflow (axial) direction (Saric 1994). At $R e=2700$, these perturbations are damped and do not affect the basic state in the long-time limit. When $R e>R e_{\text {crit }}$, these perturbations result in an unsteady nonlinear flow.

\section{Comparison between linear modes and nonlinear periodic solutions}

Of the three experimentally observed unsteady branches of solutions, only two have time-periodic axisymmetric components. We begin by examining the periodic solutions on branch 1 at $R e=3000$ and on branch 3 at $R e=4000$. Figure 9 shows contours of $\gamma$ and $\psi$ over one period of each of these nonlinear solutions. The most striking features of these contours are the unsteadiness of the vortex breakdown recirculation 

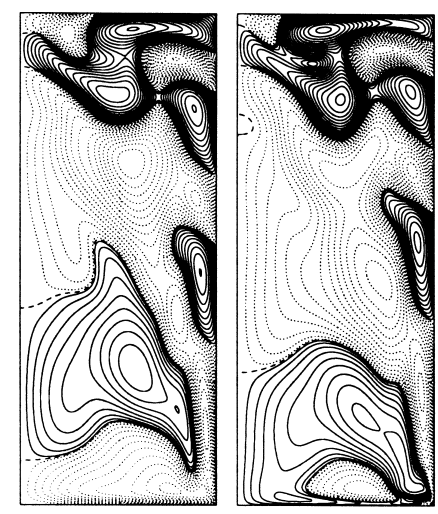

(a) $\gamma-\gamma_{b}, R e=4000$
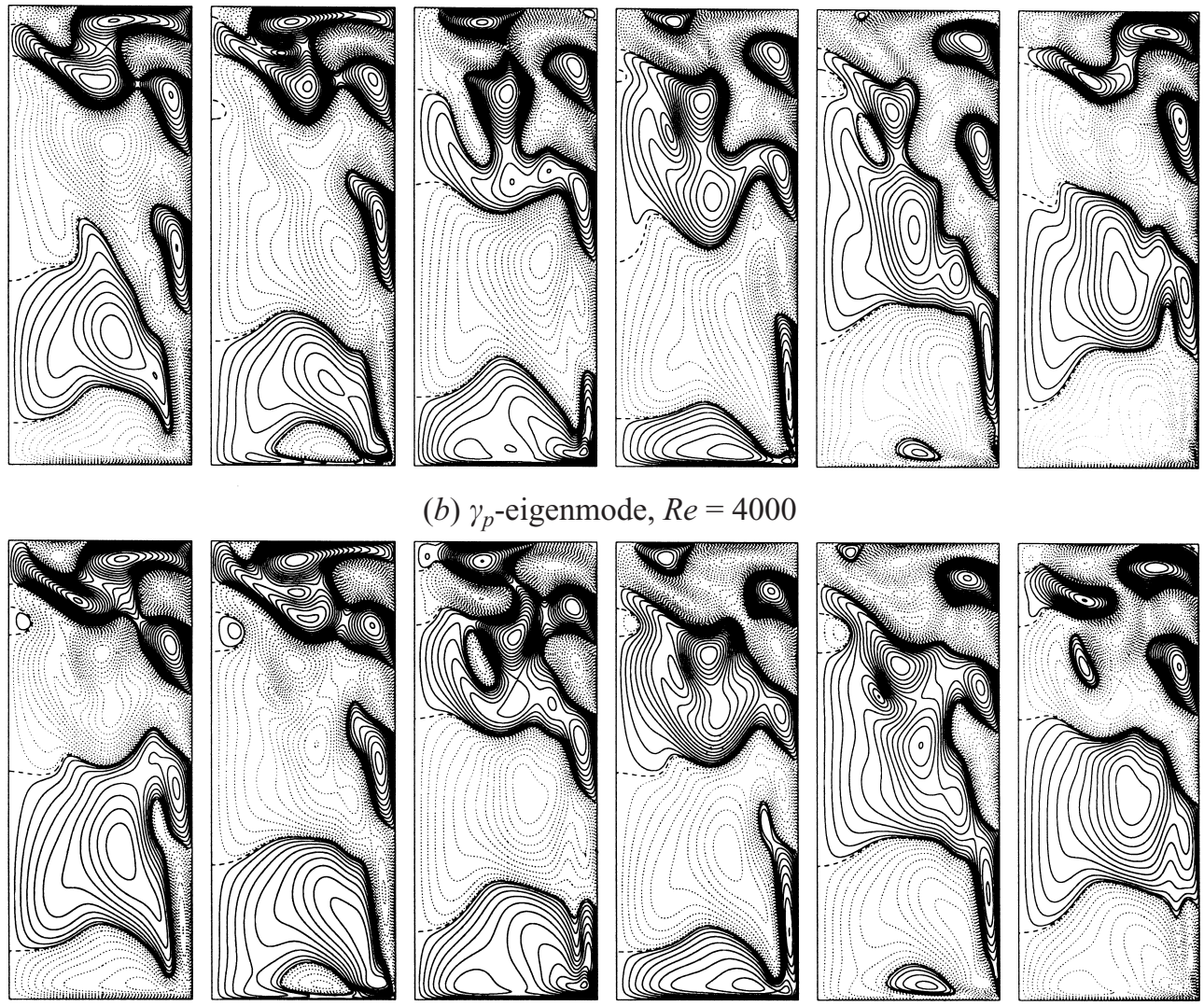

(b) $\gamma_{p}$-eigenmode, $R e=4000$
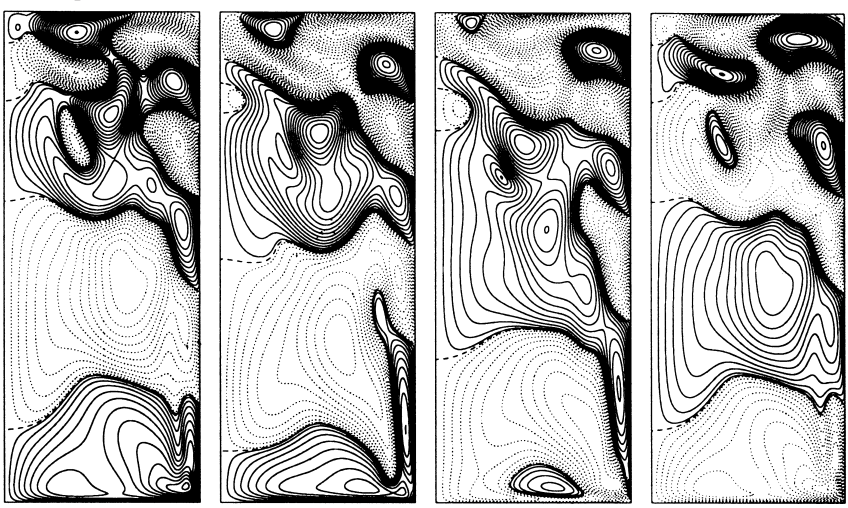

FiguRE $12(a, b)$. For caption see facing page.

zone on the axis and the flickering at the tip of the angular momentum jet. On branch 1 the unsteadiness consists of a pulsation of the recirculation zone whereas on branch 3 the recirculation zone forms and travels down the axis periodically. These two nonlinear solution branches have been described in detail in Lopez \& Perry (1992).

Figure 10 shows the corresponding steady basic states $\left(\gamma_{b}, \psi_{b}\right)$. The nonlinear perturbation fields, $\left(\gamma-\gamma_{b}, \psi-\psi_{b}\right)$, for $R e=3000$ are shown in figures $11(a)$ and $11(c)$. These differences are compared with the $\left(\gamma_{p}, \psi_{p}\right)$-eigenmode corresponding to the most dangerous eigenvalue at $R e=3000, \lambda_{1}$ (the only eigenvalue with positive real part), shown in figures $11(b)$ and $11(d)$. The linear eigenmode captures all the spatial structure of the nonlinear perturbation. This is not unreasonable since the Hopf bifurcation of $\lambda_{1}$ at $R e_{\text {crit }}=2707$ is supercritical, relatively close to the supercritical $R e$ considered, and there are no other eigenvalues with positive real part (excited modes).

At $R e=4000$ the basic state has three excited modes, $\lambda_{1}, \lambda_{2}$, and $\lambda_{3}$, and $\lambda_{3}$ has by far the largest real part, i.e. fastest growth rate (see figure 6). Its imaginary part, $\omega_{3}$, gives the period $2 \pi / \omega_{3}=7.175 \times 10^{-3}$ that is very close to the period of the nonlinear solution $\left(7.20 \times 10^{-3}\right)$. Furthermore, the corresponding eigenmode, shown in figures $12(b)$ and $12(d)$, also captures all the spatial structure of the nonlinear perturbation shown in figures $12(a)$ and $12(c)$. The minor differences between the eigenmode and the nonlinear perturbation can be accounted for by the fact that the 

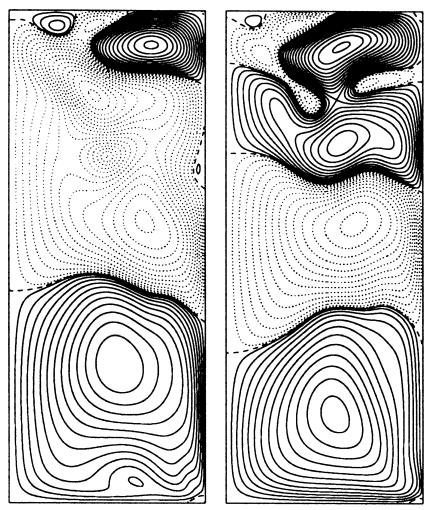

(c) $\psi-\psi_{b}, R e=4000$
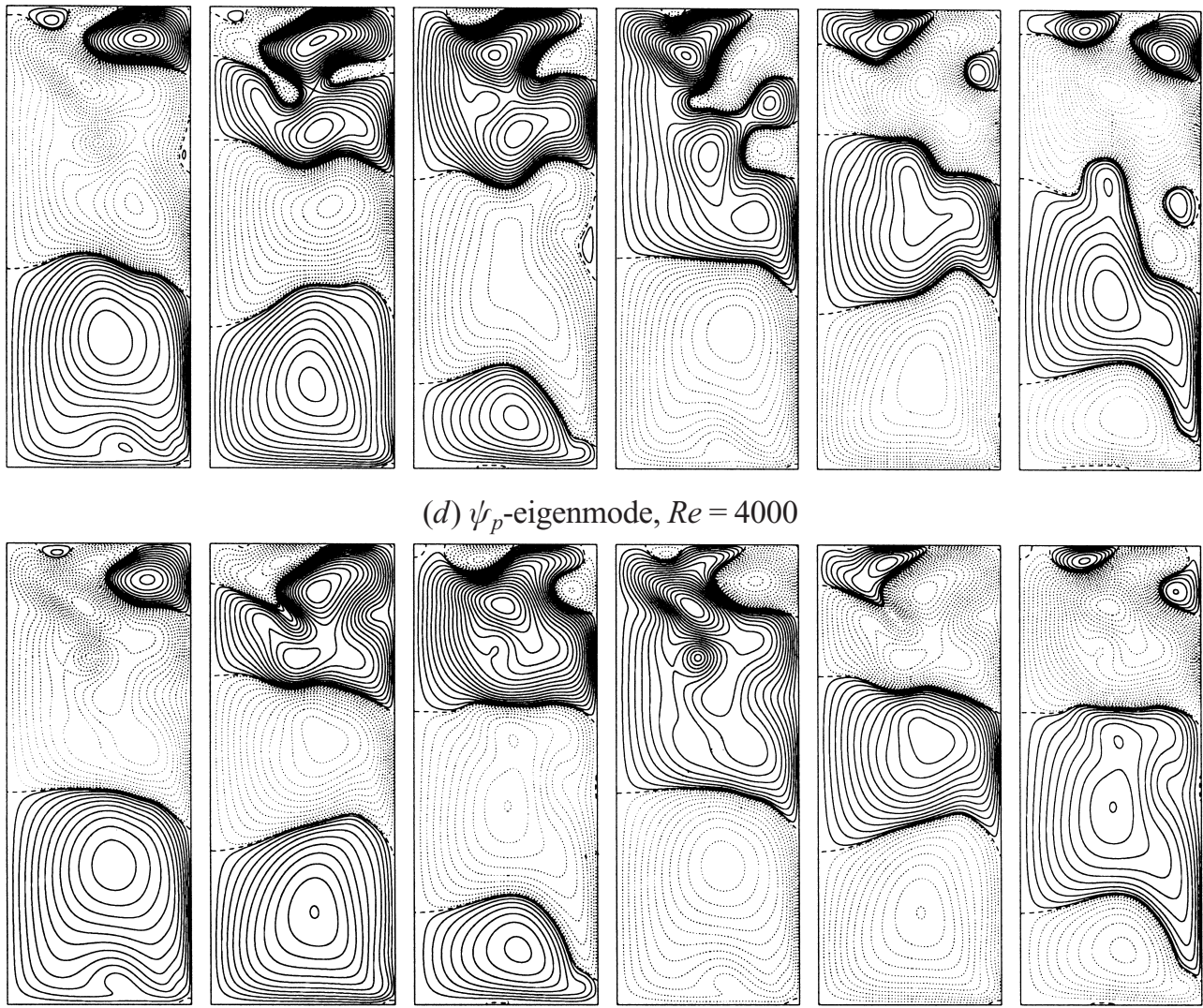

(d) $\psi_{p}$-eigenmode, $R e=4000$
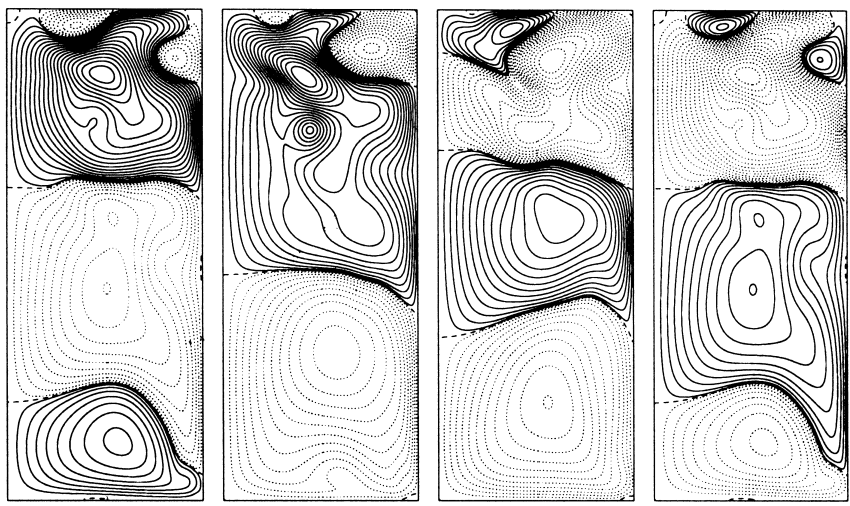

FIGURE 12. Contours of $(a) \gamma-\gamma_{b}$ and $(c) \psi-\psi_{b}$ over one nonlinear period $7.20 \times 10^{-3}$ and $(a) \gamma_{p^{-}}$ and $(c) \psi_{p}$-eigenmodes of $\lambda_{3}$ over one period $\tau_{3}=2 \pi / \omega_{3}=7.175 \times 10^{-3}$ for $R e=4000, \Lambda=2.5$, $n_{r}=140$.

linear mode has a harmonic temporal dependence whereas the nonlinear solution has significant harmonics; these are reported in Lopez \& Perry (1992) and Stevens et al. (1999) where power spectral densities (PSD) of solutions on this branch show peaks at the fundamental and several harmonics. The $\lambda_{3}$ mode bifurcates from the basic state at $R e \approx 3150$ and the resulting limit cycle is unstable. The agreement between the nonlinear solution and the linear mode so far from the bifurcation point and in the presence of other excited modes is impressive. Furthermore, the nonlinear solution at $R e=4000$ is not even axisymmetric; it supports a rotating wave with azimuthal wavenumber $m=6$, modulated by the periodic axisymmetric mode (Blackburn \& Lopez 2000). The agreement between the linear modes and the nonlinear solutions on branches 1 and 3 is a manifestation of the important role that the unstable axisymmetric solutions bifurcating from the basic state play as organizing centres for the nonlinear dynamics.

\section{Comparison of linear modes with experimentally observed states}

The two periodic branches that are observed experimentally, branches 1 and 3 , are fully accounted for by the appropriate excited linear mode of the basic state. That the two branches are only observed over particular ranges of $R e$ can also be 


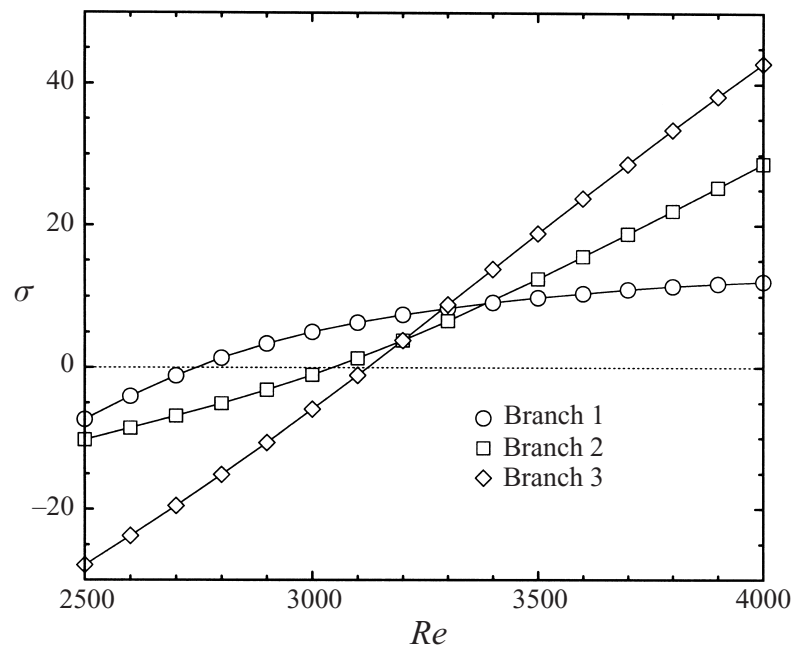

FIGURE 13. Real parts, $\sigma$, of the three most dangerous eigenvalues for $\Lambda=2.5$, versus $R e$.

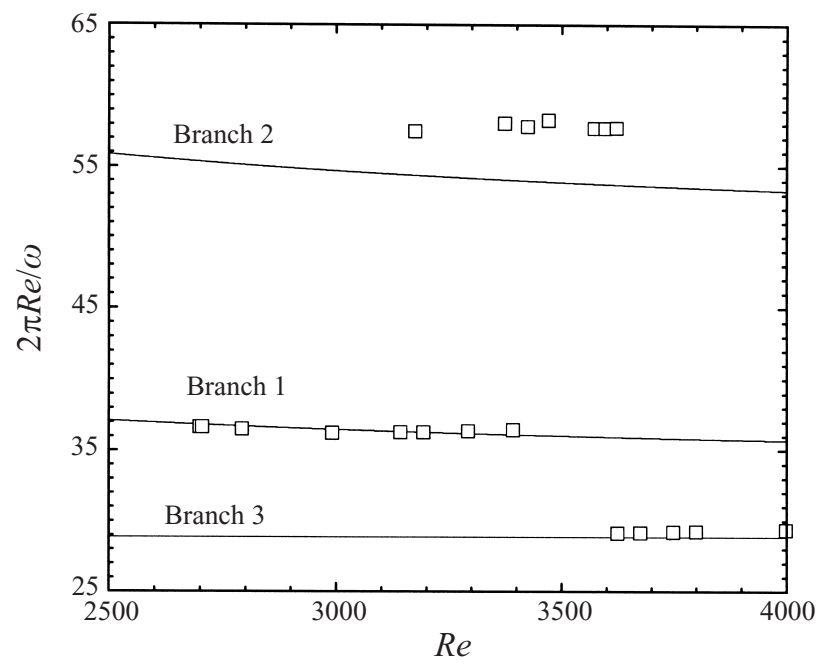

FIGURE 14. Comparison between the experimentally observed primary periods (open squares) and the periods corresponding to the three most dangerous eigenvalues (solid lines), for $\Lambda=2.5$ over a range of $R e$.

explained in terms of the growth rates of the linear modes. Figure 13 shows the growth rates of the three most dangerous eigenvalues. Notice that for $R e<3300$, $\lambda_{1}=\sigma_{1} \pm \mathrm{i} \omega_{1}$ has the largest growth rate, and that the nonlinear periodic solutions with frequency $\omega_{1}$ have been experimentally observed for $2700<R e<3400$. For $R e>3300, \lambda_{3}$ has the dominant growth rate and periodic solutions with $\omega_{3}$ have been observed for $R e>3600$. The growth rate of $\lambda_{2}$ never dominates over the $R e$ range considered, but all three growth rates are comparable for $3200<R e<3400$. The quasi-periodic solutions are observed over the range $3200<R e<3650$. This suggests the possibility that the quasi-periodic solutions are a result of a nonlinear interaction between some of the excited modes, whereas, for the periodic solutions 


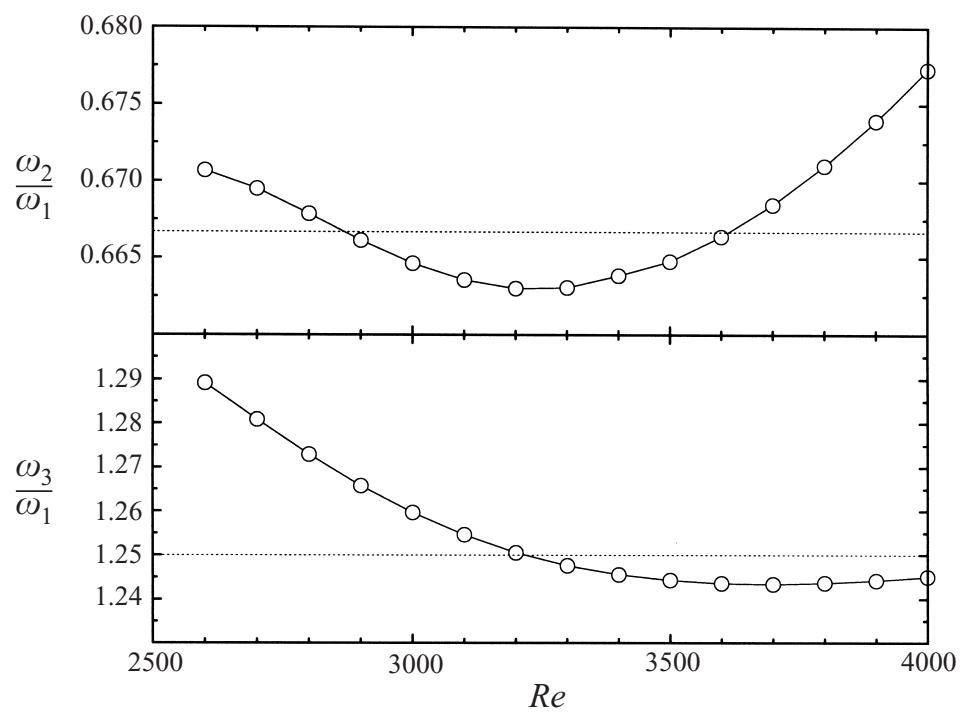

FIGURE 15. Ratios of the imaginary parts of the three most dangerous eigenvalues, $\omega_{2} / \omega_{1}$ and $\omega_{3} / \omega_{1}$, versus $R e$, together with the low-order resonances $2 / 3$ and $5 / 4$.

we have been able to associate them directly with the single most excited mode over their range of existence in $R e$.

Figure 14 shows the periods associated with each of the three most dangerous modes, scaled as $2 \pi R e / \omega$ (solid lines), together with the observed periods on branches 1 and 3 and the primary period of the quasi-periodic solutions on branch 2 (open symbols). The agreement for the periodic branches is to well within the experimental uncertainty. For the quasi-periodic solutions, the difference between the observed period and $2 \pi R e / \omega$ is about $10 \%$.

The frequencies associated with the second and third modes, $\omega_{2}$ and $\omega_{3}$, are close to low-order resonances with the frequency of the first mode. Figure 15 shows how the ratios $\omega_{2} / \omega_{1}$ and $\omega_{3} / \omega_{1}$ vary with $R e$, and that $\omega_{2} / \omega_{1}$ is close to a $2 / 3$ and $\omega_{3} / \omega_{1}$ to a $5 / 4$ resonance over an extensive range of $R e$ that includes the second and third Hopf bifurcations of the basic state. The PSD of the experimentally obtained quasi-periodic branch shows a dominant peak at $\omega_{3}$. There is another peak with comparable power at approximately $\omega_{3} / 2$, and also a low-frequency peak, $\omega_{\mathrm{lf}}$. These peaks can be explained by considering that $\omega_{2} / \omega_{3}=\left(\omega_{2} / \omega_{1}\right) /\left(\omega_{3} / \omega_{1}\right) \approx(2 / 3) /(5 / 4)=8 / 15=1 / 2+1 / 30$. The low frequency observed experimentally and numerically (Stevens et al. 1999) is very close to $\omega_{3} / 30$ and therefore the $\omega_{\text {lf }} \approx \omega_{3} / 30$ peak may result from an interaction between the $\omega_{2}$ and $\omega_{3}$ modes. In fact, $\omega_{2}-\omega_{3} / 2 \approx \omega_{3} / 30 \approx \omega_{\text {If }}$. Remember that $\lambda_{3}$ and $\lambda_{2}$ are the eigenvalues with the largest positive growth rates for $R e>3400$, so this further suggests that the mixed (quasi-periodic) state could be a combination of the two most excited modes.

\section{Conclusions}

The observed multiplicity of stable time-dependent states in the flow in a cylinder driven by a rotating endwall has been analysed in terms of the linear modes of the corresponding axisymmetric basic state. This basic state is non-trivial in the sense that it depends on both the radial and axial directions in a finite container. We have 
developed a suite of numerical techniques, exploiting the biharmonic formulation of the problem, that allows us to compute the nonlinear time evolution, the basic state, and its linear stability in a consistent and efficient manner.

Over an extensive range of $R e$ all the eigenmodes associated with eigenvalues that have positive real part are found. These excited modes of the basic state are all oscillatory. Over a similar range of $R e$, the multiple unsteady nonlinear states co-exist and are stable. By matching their frequencies to particular excited linear modes, we have been able to account for almost all of the spatial and temporal characteristics of the nonlinear states in terms of the bifurcated modes from the basic state.

The several most dangerous modes are associated with the centrifugal instability of the primary flow between the azimuthal jet and the sidewall. These instability modes are reminiscent of Görtler vortices, subjected to a drift up the wall caused by the induced secondary meridional flow associated with the basic state. The oscillations of the recirculation zones on the axis, which are of either a pulsation or travelling wave type for distinct nonlinear states, are seen to be slaved responses driven by the linear instability modes.

A full understanding, in terms of bifurcation theory, of the connection between the stable nonlinear time-dependent states and the excited modes of the basic state, particularly the modes that emerge as unstable limit cycles, requires the development of continuation techniques for unstable limit cycles and their Floquet analysis.

This work was supported by NSF grants DMS-9706951, INT-9732637 and CTS9908599 (USA), and DGICYT grants PB97-0685, PR1999-0272 (Spain).

\section{REFERENCES}

Blackburn, H. M. \& Lopez, J. M. 2000 Symmetry breaking of the flow in a cylinder driven by a rotating endwall. Phys. Fluids 12, 2698-2701.

Brown, G. L. \& Lopez, J. M. 1990 Axisymmetric vortex breakdown. Part 2. Physical mechanisms. J. Fluid Mech. 221, 533-552.

Edwards, W. S., Tuckerman, L. S., Friesner, R. A. \& Sorensen, D. C. 1994 Krylov methods for the incompressible Navier-Stokes equations. J. Comput. Phys. 110, 82-102.

EsCuDIER, M. P. 1984 Observations of the flow produced in a cylindrical container by a rotating endwall. Exps. Fluids 2, 189-196.

Gelfgat, A. Y., Bar-Yoseph, P. Z. \& Solan, A. 1996 Stability of confined swirling flow with and without vortex breakdown. J. Fluid Mech. 311, 1-36.

Gelfgat, A. Y., BAR-Yoseph, P. Z. \& Solan, A. 2001 Three-dimensional instability of axisymmetric flow in a rotating lid-cylinder enclosure. J. Fluid Mech. 438, 363-377.

Knobloch, E. 1994 Bifurcations in rotating systems. In Lectures on Solar and Planetary Dynamos (ed. M. R. E. Proctor \& A. D. Gilbert), pp. 331-372. Cambridge University Press.

LehoucQ, R. B., Sorensen, D. C. \& Yang, C. 1998 ARPACK User's Guide: Solution of Large-Scale Eigenvalue Problems with Implicitly Restarted Arnoldi Methods. SIAM.

Lopez, J. M. 1990 Axisymmetric vortex breakdown. Part 1. Confined swirling flow. J. Fluid Mech. 221, 533-552.

Lopez, J. M. \& Perry, A. D. 1992 Axisymmetric vortex breakdown. Part 3. Onset of periodic flow and chaotic advection. J. Fluid Mech. 234, 449-471.

SARIC, W. S. 1994 Görtler vortices. Ann. Rev. Fluid Mech. 26, 379-409.

Sorensen, J. N. \& Christensen, E. A. 1995 Direct numerical simulation of rotating fluid flow in a closed cylinder. Phys. Fluids 7, 764-778.

Spohn, A., Mory, M. \& Hopfinger, E. J. 1998 Experiments on vortex breakdown in a confined flow generated by a rotating disk. J. Fluid Mech. 370, 73-99.

Stevens, J. L., Lopez, J. M. \& CANTwell, B. J. 1999 Oscillatory flow states in an enclosed cylinder with a rotating endwall. J. Fluid Mech 389, 101-118. 
Trefethen, L. N. \& BAU, D. 1997 Numerical Linear Algebra. SIAM.

Tuckerman, L. S. \& BARKLeY, D. 2000 Bifurcation analysis for timesteppers. In Numerical Methods for Bifurcation Problems and Large-Scale Dynamical Systems (ed. E. Doedel \& L. S. Tuckerman). IMA Volumes in Mathematics and its Applications, vol. 119, pp. 453-466. Springer.

Vogel, H. U. 1968 Experimentelle Ergebnisse über die laminare Strömung in einen zylindrischen Gehäuse mit darin rotierender Scheibe. Tech. Rep. Bericht 6. Max-Plank-Inst. 